\title{
Efeito da quimioterapia adjuvante sobre o peso e índice de massa corporal em mulheres com câncer de mama
}

Eliana da Silva Rezende Mendes

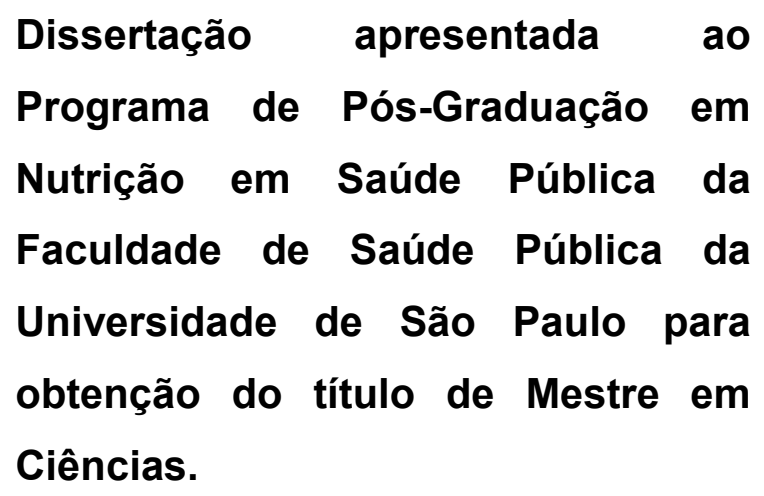

Área de Concentração: Nutrição em Saúde Pública

Orientadora: Profa. Dra. Maria de Fátima Nunes Marucci

São Paulo

2009 
É expressamente proibida a comercialização deste documento, tanto na sua forma impressa como eletrônica. Sua reprodução total ou parcial é permitida, exclusivamente, para fins acadêmicos e científicos, desde que, na reprodução, figure a identificação do autor, título, instituição e ano da tese / dissertação. 


\section{AGRADECIMENTOS}

À Deus, por ter me concedido persistência em chegar até aqui.

À minha orientadora, Prof ${ }^{\mathrm{a}}$. $\mathrm{Dr}^{\mathrm{a}}$. Maria de Fátima Nunes Marucci, pela dedicação, orientação e confiança.

Ao Dr. Auro e toda equipe da $\mathrm{CLIOH}$, por terem viabilizado minha coleta de dados e acreditado no meu trabalho.

A todas as pacientes que autorizaram a coleta de dados, possibilitando a realização deste trabalho.

À Dra. Letícia A. Vervloet e aos colegas Luis Alberto Gobbo e Manuela Ferreira de Almeida, pela ajuda na análise estatística dos dados.

À equipe do Núcleo Especializado em Oncologia (NEON), por permitirem a conclusão do mestrado.

Às minhas amigas, Juliana dos Santos Cantária e Márcia Salgueiro, pelo incentivo durante todo o curso.

Aos meus queridos amigos, que sempre acreditaram em mim e me apoiaram.

À minha família, especialmente, meus pais, Hélio e Edelta, minha sogra, D. Cleyde, meu marido e grande amor, Flávio e meus filhos, Rafael e Pedro, razões da minha vida, pelo apoio eterno, carinho e compreensão. 


\section{RESUMO}

Mendes, ESR. Efeito da quimioterapia adjuvante sobre o peso e índice de massa corporal de mulheres com câncer de mama. [Dissertação de mestrado]. São Paulo: Faculdade de Saúde Pública da Universidade de São Paulo; 2009.

Introdução: O aumento de peso corporal e o IMC entre 25 e $29,9 \mathrm{~kg} / \mathrm{m}^{2}$ (risco de obesidade) ou $\geq 30 \mathrm{~kg} / \mathrm{m}^{2}$ (obesidade), no momento do diagnóstico do câncer de mama, são fatores de risco, reconhecidos, para a recidiva da doença e redução do tempo de vida das mulheres. Objetivo: verificar os efeitos da quimioterapia adjuvante sobre o peso e índice de massa corporal de mulheres com câncer de mama. Métodos: o estudo foi descritivo, exploratório, com coleta de dados, a partir de prontuários e contato telefônico, de uma amostra de conveniência de 66 mulheres, com câncer de mama, e submetidas à quimioterapia adjuvante, no período de 2000 a 2008. As variáveis de estudo foram peso e índice de massa corporal, protocolo quimioterápico, número de ciclos de quimioterapia, fase do climatério (pré e pós-menopausa), ingestão alimentar e prática de atividade física. A análise estatística foi realizada por meio dos testes qui-quadrado, t-Student e ANOVA. Foi adotado nível de significância de 0,05 e intervalo de confiança de 95\%. Resultados: as mulheres tinham, em média, 49 anos de idade (26 - 75 anos), sendo que a alteração de peso corporal foi associada, significativamente $(p<0,05)$, com número de ciclos de quimioterapia e alteração da prática de atividade física, enquanto o protocolo quimioterápico, a fase do climatério e a ingestão alimentar não alteraram, significativamente, o peso corporal; não houve diferença, estatisticamente significante, entre IMC, no início da quimioterapia, e as demais variáveis do estudo. Conclusões: o maior o número de ciclos e a prática de atividade física foram estatisticamente significantes, com alteração do peso corporal.

Descritores: câncer de mama, peso corporal, índice de massa corporal, quimioterapia adjuvante. 


\section{ABSTRACT}

Mendes, ESR. Efeito da quimioterapia adjuvante sobre o peso e índice de massa corporal de mulheres com câncer de mama. / Effect of adjuvant chemotherapy on body weight and body mass index in women with breast cancer [dissertation]. São Paulo (BR): Faculdade de Saúde Pública da Universidade de São Paulo; 2009.

Introduction: The increase of body weight and the BMI between 25 to 29,9 $\mathrm{kg} / \mathrm{m}^{2}$ (overweight) or $\geq 30 \mathrm{~kg} / \mathrm{m}^{2}$ (obese), at the moment of breast cancer diagnosis, are factors of risk, acknowledged, for the recurrence of disease and reduction of survival. Objective: to access the effects of adjuvant chemotherapy on body weight and body mass index in women with breast cancer diagnosis. Methods: the study was descriptive, exploratory, with data collected from medical record and phone contacts with women diagnosed breast cancer, which had undergone adjuvant chemotherapy between 2000 and 2008. The study variables were body weight and body mass index, chemotherapy regimens, number of cycles of chemotherapy, menopausal status (pre and pos menopause), dietary intake and physical activity. The statistical analysis was done by qui-square, t-Student and ANOVA. The significance level adopted was 0,05 and the confidence interval was $95 \%$. Results: 66 women were analyzed, averaging 49 years old ( 26 to 75 years old), and the body weight difference was associated, significantly $(p<0,05)$, to the number of cycles of chemotherapy and changes in the physical activity practice, while the chemotherapy regimens, menopausal status and the dietary intake didn't change the body weight significantly; there was no statistically significant difference between BMI, in the beginning of chemotherapy and others variables.Conclusions: number of cycles and physical activities practice were statistically significant, with changes in body weight.

Key words: breast cancer, corporal weight, body mass index, adjuvant chemotherapy. 


\section{ÍNDICE}

1 INTRODUÇÃO 10

1.1 EPIDEMIOLOGIA DO CÂNCER DE MAMA 10

1.2 FISIOPATOLOGIA E ESTADIAMENTO DO CÂNCER

DE MAMA 11

1.3 QUIMIOTERAPIA ADJUVANTE 13

1.4 PESO CORPORAL E ÍNDICE DE MASSA CORPORAL (IMC)

1.5 FASE DO CLIMATÉRIO 16

1.6 INGESTÃO ALIMENTAR 17

1.7 PRÁTICA DE ATIVIDADE FÍSICA 19

2 OBJETIVO 19

3 MÉTODO 20

3.1. DELINEAMENTO DO ESTUDO 20

3.2. POPULAÇÃO DO ESTUDO 20

3.2.1. Critérios de inclusão 20

3.2.2. Critérios de exclusão 20

3.3. ASPECTOS ÉTICOS 20

3.4. VARIÁVEIS DO ESTUDO 21

3.5. ANÁLISE DOS DADOS 23

4 RESULTADOS 24

4.1 CARACTERIZAÇÃO DA POPULAÇÃO DE ESTUDO 24

4.2 ALTERAÇÃO DE PESO E IMC 28

4.2.1 Quimioterapia Adjuvante 28

4.2.2 Fase do Climatério 32

4.2.3 Ingestão Alimentar $\quad 35$

4.2.4 Prática de Atividade Física 38

5 DISCUSSÃo 42 
6 CONCLUSÒES 52

7 REFERÊNCIAS 53

ANEXOS

Anexo 1 - Termo de consentimento livre e esclarecido 60

Anexo 2 - Ficha para coleta dos dados 62

Anexo 3 - Parecer do Comitê de Ética 63 


\section{Lista de Tabelas}

Tabela 1 - Distribuição das mulheres, segundo grupo etário, características clínicas, alteração de peso corporal e IMC

Tabela 2 - Variação e valores médios de peso corporal e IMC das mulheres

Tabela 3 - Distribuição das mulheres, segundo alteração de peso corporal, de acordo com classificação do IMC, no início da quimioterapia

Tabela 4 - Distribuição das mulheres, de acordo com alteração de peso corporal e protocolos de quimioterapia

Tabela 5 - Variação do peso corporal, segundo protocolos de quimioterapia

Tabela 6 - Distribuição das mulheres, segundo classificação do IMC, no início da quimioterapia, e protocolos de quimioterapia

Tabela 7 - Distribuição das mulheres, segundo alteração de peso corporal, de acordo com número de ciclos de quimioterapia

Tabela 8 - Variação do peso corporal, de acordo com o número de ciclos de quimioterapia

Tabela 9 - Distribuição das mulheres, segundo classificação do IMC, no início da quimioterapia, e número de ciclos de quimioterapia

Tabela 10 - Distribuição das mulheres, de acordo com alteração de peso corporal e fase do climatério

Tabela 11 - Variação do peso corporal, de acordo com a fase do climatério

Tabela 12 - Distribuição das mulheres, segundo classificação do IMC, no início da quimioterapia, e fase do climatério

Tabela 13 - Distribuição das mulheres, de acordo com alteração de peso corporal e da ingestão alimentar

Tabela 14 - Variação do peso corporal, de acordo com a ingestão Alimentar 
Tabela 15 - Distribuição das mulheres, segundo classificação do IMC, no início da quimioterapia, e ingestão alimentar

Tabela 16 - Distribuição das mulheres de acordo com alteração de peso corporal e prática de atividade física

Tabela 17 - Variação do peso corporal, de acordo com a prática de atividade física atividade física

Tabela 18 - Distribuição das mulheres, segundo classificação do IMC, no início da quimioterapia, e prática de atividade física 


\subsection{INTRODUÇÃO}

\subsection{EPIDEMIOLOGIA DO CÂNCER DE MAMA}

O câncer, devido à sua elevada incidência e ao aumento da expectativa de vida ao nascer, é considerado um dos mais importantes problemas de saúde pública do mundo (PAULINELLE et al., 2003; CLARKE et al., 2006, KIRJNER e PINHEIRO, 2007).

Em 2005, foi responsável por 7,6 milhões (13\%) de mortes, sendo mais de $70 \%$, nos países em desenvolvimento. Estima-se que, em 2020, o número de casos novos anuais seja de, aproximadamente, 15 milhões, $60 \%$ deles, em países em desenvolvimento (INCA, 2007).

Segundo a Organização Mundial da Saúde (OMS), estima-se mais de 1.050.000 de casos novos de câncer de mama em todo o mundo, por ano. Além disso, esse câncer é a causa mais freqüente de morte em mulheres com menos de 50 anos de idade (INCA, 2006).

No Brasil, ele é o segundo mais incidente e, as estimativas para o ano de 2008 e 2009, são de 49.400 casos novos, sendo que a região Sudeste será a mais incidente, com um risco estimado de 68 casos novos por 100 mil mulheres (INCA, 2007).

Os fatores de risco para o câncer de mama estão relacionados à: fase reprodutiva da mulher (menarca precoce - antes dos 12 anos de idade, menopausa tardia - após 55 anos de idade, nuliparidade, idade da primeira gestação a termo superior a 30 anos, uso de anticoncepcionais orais e de terapia de reposição hormonal), idade (o risco aumenta após os 50 anos), etnia (mulheres afro-descendentes tem maior probabilidade de desenvolver a doença do que mulheres brancas), história familiar (parente de primeiro grau, com câncer de mama ou de ovário), história pessoal (mulheres que tiveram câncer em uma das mamas, tem mais chance de desenvolver na outra), predisposição 
genética (mutações nos genes BRCA1 ou BRCA2 estão associadas ao aumento do risco da doença), fumo, estilo de vida (ingestão alimentar inadequada, sedentarismo, obesidade e ingestão de bebidas alcoólicas) (KELSEY et al., 1988; BARROS et al., 2001; THULER et al., 2003; PAULINELLE et al., 2003; INCA, 2007).

Avanços na detecção precoce e no tratamento do câncer de mama proporcionaram aumento do tempo de vida das mulheres, sendo que, nos Estados Unidos, aproximadamente 2,3 milhões, são consideradas sobreviventes dessa doença (KUSHI et al., 2007).

\subsection{FISIOPATOLOGIA E ESTADIAMENTO DO CÂNCER DE MAMA}

O termo câncer é a tradução latina da palavra grega carcinoma (karkinos = crustáceo, caranguejo), e é usado para indicar qualquer neoplasia maligna (BRASILEIRO FILHO et al., 2006).

Entre os diferentes tumores malignos da mama, os carcinomas são os mais importantes (SCHMITT e GOBBI, 2006).

Clinicamente, o carcinoma da mama manifesta-se como nódulo palpável, muitas vezes detectável pela própria mulher, por meio de anormalidades mamográficas, como achado incidental histológico em fragmentos de mama retirados por outra razão (doença benigna ou mamoplastia), pelo encontro de células malignas na investigação de derrames papilares ou por suas metástases (SCHMITT e GOBBI, 2006).

O câncer de mama pode ser classificado em carcinoma in situ, os quais estão localizados nos ductos ou lóbulos e, não se propagam para os tecidos circunvizinhos, ou invasivos (ou infiltrativos), que penetram nos ductos e lóbulos, e invadem os tecidos circunvizinhos (SCHMITT e GOBBI, 2006).

O estadiamento do câncer de mama é feito por meio de critérios estabelecidos pela União Internacional Contra o Câncer (UICC). Ele se baseia na classificação TNM, em que é verificado o tamanho do tumor (T), existência 
de metástases nos linfonodos regionais $(\mathrm{N})$ e metástases à distância $(\mathrm{M})$ e só se aplica aos carcinomas, os quais representam $80 \%$ dos tumores mamários (INCA 2004a e 2004b).

Quadro 1 - Agrupamento das variáveis T, N e M em estádios, de acordo com os critérios da UICC.

\begin{tabular}{|c|c|}
\hline Estadio & Agrupamento \\
\hline Estadio 0 & TisN0M0 \\
\hline Estadio I & T1N0M0 \\
\hline Estadio II A & T0N1M0 \\
& T1N1M0 \\
& T2N0M0 \\
\hline Estadio II B & T2N1M0 \\
\hline & T3N0M0 \\
\hline & T0N2M0 \\
\hline & T1N2M0 \\
& T2N2M0 \\
& T3N1M0 \\
& T3N2M0 \\
\hline Estadio III A & T4N0M0 \\
\hline Estadio III B & T4N1M0 \\
\hline & T4N2M0 \\
\hline & TqqN3M0* \\
\hline & TqqNq9M1* \\
\hline
\end{tabular}

${ }^{*} \mathrm{qq}=$ qualquer

Fonte: INCA, 2004b. 


\subsection{QUIMIOTERAPIA ADJUVANTE}

A quimioterapia adjuvante é utilizada após a ressecção completa do tumor, quando não há evidência de metástase à distância (GIMENES, 2003; BONASSA, 2005). Está recomendada à maioria das mulheres com doença localizada e proporciona redução de recidiva em cerca de 30\% (DEL GIGLIO e SAMANO, 2004).

Os protocolos quimioterápicos variam de acordo com o tipo, estadio do câncer e das condições clínicas do paciente. As doses dos medicamentos são calculadas com base na superfície corporal total, expressa em metros quadrados $\left(\mathrm{m}^{2}\right)$, sendo que a administração é feita com a dose mais alta tolerada (DEL GIGLIO e SAMANO, 2004; KIRJNER e PINHEIRO, 2007).

De modo geral, a quimioterapia adjuvante é realizada em ciclos que podem ser constituídos de um ou vários dias de aplicação. Cada ciclo é repetido dentro de um intervalo mínimo de tempo (para câncer de mama, o intervalo é, normalmente, a cada 21 ou 28 dias), para que o organismo se recupere dos efeitos colaterais, que variam de acordo com os medicamentos utilizados (GIMENES, 2003; DEL GIGLIO e SAMANO, 2004).

Os efeitos colaterais da quimioterapia estão relacionados à toxicidade de cada medicamento que compõe o protocolo quimioterápico. Os mais comuns são náusea, vômito, alopécia, mielotoxicidade, mucosite, constipação intestinal, diarréia, redução ou aumento de peso corporal, toxicidade pulmonar, cardíaca e renal (GIMENES, 2003).

Sempre que possível, procura-se combinar vários medicamentos que sejam ativos contra um tumor, em um protocolo poliquimioterápico (com mais de duas drogas), uma vez que, essa combinação é mais eficaz do que cada medicamento administrado separadamente (GIMENES, 2003; DEL GIGLIO e SAMANO, 2004).

De acordo com PINDER et al. (2007), as antraciclinas estão entre as drogas mais efetivas para o tratamento de mulheres com câncer de mama, 
sendo que a poliquimioterapia adjuvante com antraciclinas reduz a taxa de mortalidade em $38 \%$ em mulheres de até 50 anos de idade e, em $20 \%$ nas mulheres com idade superior a 50 anos. Além disso, aumentam, em $3 \%$, o tempo de vida em 5 anos e em 4\%, o tempo de vida em 10 anos.

Neste sentido, há fortes evidências de que protocolos quimioterápicos contendo antraciclinas (doxorrubicina e ciclofosfamida - AC; fluorouracil, doxorrubicina e ciclofosfamida - FAC e fluorouracil, epirrubicina e ciclofosfamida - FEC), são mais eficazes do que aqueles sem antraciclinas (ciclofosfamida, metotrexato e fluorouracil - CMF) (AAPRO, 2001).

As características dos medicamentos que compõem os protocolos com e sem antraciclinas estão apresentadas no Quadro 2.

Quadro 2 - Características e principais efeitos colaterais dos medicamentos utilizados nos protocolos com e sem antraciclinas.

\begin{tabular}{|c|c|c|}
\hline Medicamentos & Características & Efeitos colaterais \\
\hline Ciclofosfamida & Agente alquilante ${ }^{a}$ & Náusea, vômito, diarréia, dor abdominal, etc. \\
\hline Fluorouracil & Antimetabólito ${ }^{\mathrm{b}}$ & Náusea, vômito, estomatites, diarréia, etc. \\
\hline $\begin{array}{c}\text { Doxorrubicina / } \\
\text { Epirrubicina }\end{array}$ & $\begin{array}{c}\text { Antibiótico antitumoral } \\
\text { antracíclico }^{c}\end{array}$ & $\begin{array}{l}\text { Anemia, anorexia, desidratação, náusea, } \\
\text { vômito, diarréia, mucosite, etc. }\end{array}$ \\
\hline Metotrexato & Antimetabólito ${ }^{\mathrm{b}}$ & Náusea, vômito, mucosite, anorexia, etc. \\
\hline
\end{tabular}

Fonte: GIMENES (2003); DEL GIGLIO e SAMANO (2004); BONASSA (2005).

a: Capazes de quebrar a sequência da molécula de DNA, impedindo sua replicação.

${ }^{\mathrm{b}}$ : Inibidores da síntese de DNA.

c: Impedem a duplicação e separação das cadeias de DNA e RNA.

d: Ocasionam bloqueio da divisão celular 


\subsection{PESO CORPORAL E IMC}

Numerosos autores têm constatado que mulheres submetidas à quimioterapia adjuvante apresentam aumento de peso corporal durante o tratamento (GOODWIN et al., 1999; ROONEY e WALD, 2007).

O aumento de peso corporal e o IMC entre 25 e $29,9 \mathrm{~kg} / \mathrm{m}^{2}$ (risco para obesidade) ou $\geq 30 \mathrm{~kg} / \mathrm{m}^{2}$ (obesidade), no momento do diagnóstico do câncer de mama, são fatores de risco, reconhecidos, para a recidiva da doença e redução do tempo de vida das mulheres (GOODWIN et al., 2003; ROONEY e WALD, 2007).

O câncer de mama é uma doença hormônio-dependente, fortemente influenciada pelos hormônios esteróides gonadais e a estimulação estrogênica está envolvida com a patogênese da doença, uma vez que os hormônios ovarianos são promotores tumorais (ROCK, 2003).

Por outro lado, a obesidade é associada ao aumento da concentração de estrona e estradiol em mulheres pós-menopausadas e à redução dos níveis de globulina ligada aos hormônios sexuais, resultando no aumento da fração biodisponível de estrógeno e, por conseguinte, aumento do risco de recidiva da doença e redução do tempo de vida das mulheres (ROCK e DEMARKWAHNEFRIED, 2002).

Em estudo de revisão feito por ROCK e DEMARK-WAHNEFRIED (2002), os autores verificaram associação, estatisticamente significante, entre aumento do peso corporal e do IMC com o risco de recidiva da doença, em 17 dos 26 estudos analisados. CHLEBOWISK et al. (2002) também constataram associação, estatisticamente significante, em 26 de 34 estudos revisados sobre obesidade, recidiva do câncer de mama e tempo de vida das mulheres.

BERCLAZ et al. (2004) avaliaram 6792 mulheres com câncer de mama e constataram que o risco de recidiva e morte foi maior para as mulheres obesas (17\% e $14 \%$, respectivamente) e que o IMC foi fator prognóstico independente para tempo de vida das mesmas. 
BASTARRACHEA et al. (1994) analisaram 735 mulheres e verificaram que, após 10 anos de acompanhamento, as mulheres que apresentaram aumento maior de $20 \%$ do peso corporal inicial, tiveram tempo de vida sem recidiva, significativamente menor, do que aquelas que apresentaram valor médio de aumento de peso corporal menor de $20 \%$.

O aumento de peso corporal em mulheres com câncer de mama durante a quimioterapia está associado à poliquimioterapia sem antraciclinas, ao maior número de ciclos da quimioterapia, à pré-menopausa, ao aumento da ingestão alimentar e à redução da prática de atividade física (DEMARK-WAHNEFRIED et al., 1993 e 1997; CHLEBOWISK et al., 2002; FREEDMAN et al., 2004).

Além disso, ele ocasiona impacto negativo na auto-estima das mulheres com essa doença, sendo que 20 a $60 \%$ referem insatisfação com seu corpo após o diagnóstico, o que contribui para depressão, piorando o prognóstico das mesmas (PINTO e TRUNZO, 2004).

\subsection{FASE DO CLIMATÉRIO}

Muitos autores têm constatado que mulheres pré-menopausadas apresentam maior valor médio de aumento de peso corporal, durante a quimioterapia adjuvante (DEMARK-WAHNEFRIED et al., 1993 e 1997; GOODWIN et al., 1999; COSTA et al., 2002; LANKESTER et al., 2002).

Esse tratamento ocasiona redução da função ovariana, submetendo as mulheres pré-menopausadas à menopausa prematura (CAMORIANO et al., 1990; DEMARK-WAHNEFRIED et al., 1993; KUMAR et al., 2004).

POEHLMAN e TCHERNOF (1998) concluíram que a interrupção da função ovariana ocasiona redução do metabolismo, da quantidade de massa muscular, e do gasto energético na atividade física, além de estimular o acúmulo de gordura no tecido adiposo.

CAMORIANO et al. (1990) avaliaram 545 mulheres com câncer de mama durante a quimioterapia adjuvante e verificaram que as pré-menopausadas 
apresentaram maior valor médio de aumento de peso corporal, do que as pósmenopausadas $(5,9$ e 3,6 kg, respectivamente; $p<0,001)$.

GOODWIN et al. (1999) constataram que mulheres que ficaram menopausadas durante a quimioterapia, apresentaram maior valor médio de peso corporal do que aquelas que permaneceram pré-menopausadas $(2,7 \mathrm{~kg} \mathrm{e}$ $0,7 \mathrm{~kg}$, respectivamente; $p=0,002$ )

De acordo com LIMA-JÚNIOR et al. (2000), mulheres, na perimenopausa, tendem a aumentar o peso corporal, numa média de 0,8 kg/ano, sendo que a mudança do hábito alimentar e o sedentarismo, são fatores de risco envolvidos.

\subsection{INGESTÃO ALIMENTAR}

Muitos autores têm avaliado a relação da ingestão alimentar com aumento de peso corporal relacionado a quimioterapia e ao tempo de vida de mulheres com câncer de mama (CHLEBOWISKI et al., 2006; PIERCE et al., 2007).

O aumento da ingestão alimentar, durante a quimioterapia adjuvante, está relacionado a tentativa de reduzir a náusea, que é um dos efeitos colaterais mais comuns do tratamento (DEMARK-WAHNEFRIED et al. 1997).

Apesar da alguns autores não demonstrarem associação entre redução do peso corporal e alimentação com baixo teor de alimentos gordurosos (ROCK et al., 2001; THOMSON et al., 2005; HOWARD et al., 2006), outros têm sugerido que alimentação equilibrada, com ingestão diária de frutas, verduras, legumes, cereais integrais e carne branca (frango e peixe), está positivamente associada ao aumento do tempo de vida de mulheres com câncer de mama (GOODWIN et al., 2003; KROENKE et al., 2005; McELIGOT et al., 2006; KWAN et al., 2008).

ROCK E DEMARK-WAHNEFRIED (2002) revisaram a literatura sobre a relação entre ingestão de alimentos com alto teor de gordura e recidiva do 
câncer de mama e verificaram associação inversa e significativa, em cinco dos 12 estudos analisados. Em oito estudos que avaliaram a ingestão de verduras e legumes, três constataram associação inversa com risco de morte (20 a 90\% de redução) e nenhum dos sete estudos que analisaram o efeito da fibra alimentar com risco de recidiva e tempo de vida verificou associação significativa.

A gordura alimentar, assim como a obesidade, é relacionada ao aumento dos níveis de estrógeno, e consequentemente, ao aumento do risco de recidiva da doença (ROCK et al., 2003).

WU et al. (1999), revisaram a literatura e constataram que a redução da ingestão de alimentos com alto teor de gordura pode reduzir a concentração de estrógeno. Entretanto os níveis desse hormônio também são reduzidos com a redução do peso corporal e com o aumento da ingestão de alimentos com alto teor de fibra.

ROCK et al. (2004) avaliaram 291 mulheres com câncer de mama e verificaram que, alimentação com alto teor de fibras (ingestão de verduras e legumes, 5 vezes ao dia, de frutas, 3 vezes ao dia e $30 \mathrm{~g}$ de fibras/dia) e baixo teor de gordura (15 a $20 \%$ da energia potencial dos alimentos), foi associada com redução, estatisticamente significante $(p<0,05)$, da concentração de estradiol biodisponível, sendo que o aumento da ingestão de alimentos com alto teor de fibras foi, independentemente associado $(p<0,05)$, à redução da concentração de estradiol sérico. Nesse estudo, as mulheres não apresentaram redução do peso corporal, nem alteração da prática de atividade física.

Muitos autores que estudam sobre alimentação em mulheres com câncer de mama, recomendam redução na ingestão de alimentos com alto teor de gordura e aumento na ingestão de frutas, verduras, legumes e cereais integrais, uma vez que essas mulheres estão mais motivadas a realizar mudanças de ingestão alimentar após o diagnóstico da doença (DEMARK-WAHNEFRIED et al., 2000; ROCK, 2003; GOODWIN et al., 2003; KROENKE et al., 2005; PIERCE et al., 2007). 


\subsection{PRÁTICA DE ATIVIDADE FÍSICA}

A redução da prática de atividade física que ocorre nas mulheres com câncer de mama, durante e após a quimioterapia adjuvante, favorece o aumento do peso corporal e o risco de recidiva da doença (DEMARKWAHNEFRIED et al., 1993; DEMARK-WAHNEFRIED et al., 1997; IRWIN et al., 2003 e 2004).

HOLMES et al. (2005) e HOLICK et al. (2008) verificaram que mulheres que praticaram atividade física após o diagnóstico de câncer de mama, apresentaram menor risco de morte por essa doença.

A atividade física tem sido associada com menores níveis de hormônios ovarianos circulantes e menores níveis de gordura corporal, que poderiam explicar o maior tempo de vida de mulheres com câncer de mama. Além disso, é relacionada à redução da fadiga, melhora da auto-estima e da qualidade de vida (PINTO e TRUNZO, 2004; IRWIN, 2009).

Apesar disso, apenas $28 \%$ das mulheres com câncer de mama, praticam atividade física durante a quimioterapia e $32 \%$ seguem a recomendação da American Cancer Society (ACS), de 150 minutos por semana de atividade moderada, após o término do tratamento (IRWIN et al., 2004).

\subsection{OBJETIVO}

Verificar os efeitos da quimioterapia adjuvante sobre o peso e índice de massa corporal, em mulheres com câncer de mama, segundo protocolo quimioterápico, número de ciclos de quimioterapia, fase do climatério, ingestão alimentar e prática de atividade física. 


\subsection{MÉTODO}

\subsection{DELINEAMENTO DO ESTUDO}

O estudo é descritivo exploratório.

\subsection{POPULAÇÃO DO ESTUDO}

Trata-se de amostra de "conveniência", de mulheres com câncer de mama, submetidas a tratamento clínico e químico (quimioterapia adjuvante), voluntárias, em clínica particular do município de São Paulo, no período de 2000 a 2008.

3.2.1 Critérios de inclusão:

- idade $\geq 20$ anos,

- tratamento com quimioterapia adjuvante.

3.2.2 Critérios de exclusão:

- diagnóstico de metástase,

- diagnóstico de hipo ou hipertireoidismo,

- uso de medicamentos com efeitos adversos sobre peso corporal: corticosteróides (em uso contínuo) e antidepressivos (tricíclicos e os inibidores seletivos da recaptação de serotonina).

\subsection{ASPECTOS ÉTICOS}

O projeto foi aprovado pelo Comitê de Ética da Faculdade de Saúde Pública da Universidade de São Paulo (Anexo 3). 


\subsection{VARIÁVEIS DO ESTUDO}

- Idade

Esse dado foi obtido do prontuário.

- Peso corporal

A mensuração do peso corporal foi realizada por enfermeiras e os valores foram registrados em prontuários, dos quais foram obtidos os dados, incluindo os valores de peso, antes do primeiro ciclo de quimioterapia (ou, até um mês depois), e após o último ciclo de quimioterapia (ou, até um mês antes).

- Índice de Massa Corporal (IMC)

O IMC foi calculado a partir da relação entre peso corporal (em quilos) e estatura (em metros) ao quadrado.

Para as mulheres de 20-60 anos, utilizou-se a classificação da Organização Mundial da Saúde (OMS, 1995):

IMC $<18,5 \mathrm{~kg} / \mathrm{m}^{2}=$ baixo peso para a estatura

$18,5<\mathrm{IMC}<24,9 \mathrm{~kg} / \mathrm{m}^{2}$ = peso adequado para a estatura

$25<\mathrm{IMC}<29,9 \mathrm{~kg} / \mathrm{m}^{2}=$ excesso de peso para a estatura (risco para obesidade)

IMC $\geq 30 \mathrm{~kg} / \mathrm{m}^{2}=$ excesso de peso para a estatura (obesidade).

Para as mulheres $\geq 60$ anos, utilizaram-se os valores sugeridos pela Organização Pan-Americana de Saúde (OPAS, 2001):

$\leq 23 \mathrm{~kg} / \mathrm{m}^{2}=$ baixo peso para a estatura

$23<\mathrm{IMC}<28 \mathrm{~kg} / \mathrm{m}^{2}=$ peso adequado para a estatura 
$28<\mathrm{IMC}<30 \mathrm{~kg} / \mathrm{m}^{2}=$ excesso de peso para a estatura (risco para obesidade)

$I M C \geq 30 \mathrm{~kg} / \mathrm{m}^{2}=$ excesso de peso para a estatura (obesidade).

- Protocolo quimioterápico adjuvante

Com antraciclina:

- adriamicina e ciclofosfamida (AC),

- fluorouracil, adriamicina e ciclofosfamida (FAC).

Sem antraciclina:

- ciclofosfamida, metotrexato e fluorouracil (CMF).

- Número de ciclos de quimioterapia:

4,6 ou $\geq 6$ ciclos.

- Fase do climatério

A classificação das mulheres, em relação à fase do climatério (pré ou pós-menopausa), foi realizada pelos médicos, sendo que foram consideradas pré-menopausadas aquelas com idade inferior a 55 anos, sem sintomas climatéricos, ou com presença de menstruação.

- Ingestão alimentar

As mulheres foram questionadas, por meio telefônico, sobre alteração da ingestão alimentar, em relação ao aumento, redução ou manutenção, durante o período de quimioterapia. 
- Prática de atividade física

As mulheres foram questionadas, por meio telefônico, sobre alteração da prática de atividade física, em relação ao aumento, redução ou manutenção, durante o período de quimioterapia.

Todos os dados foram coletados pela autora do trabalho e registrados em formulário específico (Anexo 2).

\subsection{ANÁLISE DOS DADOS}

A análise estatística descritiva das variáveis foi realizada, com auxílio do programa EPI INFO (versão 6.0) e STATA (versão 9.0).

A comparação entre as variáveis de estudo e alteração de peso corporal foi verificada pelo teste qui-quadrado $\left(\chi^{2}\right)$ e a comparação entre os valores médios de peso corporal, antes e após a quimioterapia, foi realizada pelo teste t-Student e análise de variância (ANOVA).

O nível de significância estatística adotado foi 0,05 , com intervalo de confiança de $95 \%$. 


\subsection{RESULTADOS}

\subsection{CARACTERIZAÇÃO DA POPULAÇÃO DE ESTUDO}

Foram estudadas 66 mulheres. A partir de 113 prontuários e, de acordo com os critérios de inclusão/exclusão, 93 foram consideradas elegíveis, sendo que vinte e sete (27) recusaram-se a participar do estudo (perda de 29\%).

Todas as mulheres estão vivas, sendo que aquelas que não devolveram o Termo de Consentimento assinado foram recusadas a participar do estudo. Ressalta-se que este documento deveria ser devolvido pelo correio, em envelope selado, pago pela pesquisadora. As principais características destas mulheres são: média de idade de 47 anos e, a maioria (56\%) foi submetida a seis ciclos de quimioterapia com antraciclinas (74\%), estava na pré-menopausa (74\%), apresentou aumento do peso corporal $(70 \%)$, foi classificada com peso adequado (52\%), no início do tratamento, aumentou a ingestão alimentar $(63 \%)$ e reduziu a prática de atividade física (100\%), durante a quimioterapia.

Com relação às características das mulheres que participaram do estudo, a média de idade foi 49 anos (26 - 75 anos, desvio padrão-dp $=10,76 \mathrm{e}$ mediana $=47$ anos). A maioria (74\%) tinha idade entre 40 e 59 anos, estava com doença no estadio II (67\%), apresentou receptores hormonais positivos para estrógeno e progesterona $(77 \%)$, foi submetida a seis ciclos $(79 \%)$ de quimioterapia com antraciclinas $(74 \%)$, estava na pré-menopausa $(65 \%)$, apresentou aumento do peso corporal $(68 \%)$, foi classificada com peso adequado (51\% das mulheres com idade inferior a 60 anos e $6 \%$ com mais de 60 anos), no início do tratamento, aumentou a ingestão alimentar (53\%) e reduziu a prática de atividade física (85\%), durante a quimioterapia (Tabela 1). 
Tabela 1 - Distribuição das mulheres, segundo grupo etário, características clínicas, alteração de peso corporal e IMC. São Paulo, 2000-2008.

\begin{tabular}{|c|c|c|c|c|}
\hline Variáveis & & & FA $^{a}$ & $\mathbf{F R}^{\mathrm{b}}$ \\
\hline Grupo etário (anos) & $\begin{array}{l}20 \text { a } 39 \\
40 \text { a } 59 \\
60 \text { a } 75\end{array}$ & & $\begin{array}{c}9 \\
49 \\
8\end{array}$ & $\begin{array}{l}14 \\
74 \\
12\end{array}$ \\
\hline Estadiamento & $\begin{array}{l}\text { I } \\
\text { II } \\
\text { III }\end{array}$ & & $\begin{array}{c}17 \\
44 \\
5\end{array}$ & $\begin{array}{c}26 \\
67 \\
7\end{array}$ \\
\hline $\begin{array}{l}\text { Receptores } \\
\text { hormonais }\end{array}$ & $\begin{array}{l}\text { Positivos } \\
\text { Negativos }\end{array}$ & & $\begin{array}{c}51 \\
3\end{array}$ & $\begin{array}{c}77 \\
5\end{array}$ \\
\hline $\begin{array}{l}\text { Protocolos } \\
\text { quimioterápicos }\end{array}$ & $\begin{array}{l}\text { Com antraciclina } \\
\text { Sem antraciclina }\end{array}$ & & $\begin{array}{l}49 \\
17\end{array}$ & $\begin{array}{l}74 \\
26\end{array}$ \\
\hline $\begin{array}{l}\text { Número de ciclos de } \\
\text { quimioterapia }\end{array}$ & $\begin{array}{l}4 \text { ciclos } \\
6 \text { ciclos } \\
\text { Mais de } 6 \text { ciclos }\end{array}$ & & $\begin{array}{c}9 \\
52 \\
5\end{array}$ & $\begin{array}{c}14 \\
79 \\
7\end{array}$ \\
\hline Fase do climatério & $\begin{array}{l}\text { Pré-menopausa } \\
\text { Pós-menopausa }\end{array}$ & & $\begin{array}{l}43 \\
23\end{array}$ & $\begin{array}{l}65 \\
35\end{array}$ \\
\hline $\begin{array}{l}\text { Alteração de peso } \\
\text { corporal (kg) }\end{array}$ & $\begin{array}{l}\text { Aumento } \\
\text { Redução } \\
\text { Manutenção }\end{array}$ & & $\begin{array}{c}45 \\
17 \\
4\end{array}$ & $\begin{array}{c}68 \\
26 \\
6\end{array}$ \\
\hline & Baixo peso & $\begin{array}{l}<60 \text { anos } \\
\geq 60 \text { anos }\end{array}$ & $\begin{array}{l}0 \\
1\end{array}$ & $\begin{array}{l}0 \\
2\end{array}$ \\
\hline & Peso adequado & $\begin{array}{l}<60 \text { anos } \\
\geq 60 \text { anos }\end{array}$ & $\begin{array}{c}34 \\
4\end{array}$ & $\begin{array}{c}51 \\
6\end{array}$ \\
\hline & Risco para obesidade & $\begin{array}{l}<60 \text { anos } \\
\geq 60 \text { anos }\end{array}$ & $\begin{array}{c}16 \\
2\end{array}$ & $\begin{array}{c}24 \\
3\end{array}$ \\
\hline & Obesidade & $\begin{array}{l}<60 \text { anos } \\
\geq 60 \text { anos }\end{array}$ & $\begin{array}{l}8 \\
1\end{array}$ & $\begin{array}{c}12 \\
2\end{array}$ \\
\hline
\end{tabular}

Continua. 
Tabela 1 - Distribuição das mulheres, segundo grupo etário, características clínicas, alteração de peso corporal e IMC. São Paulo, 2000-2008.

Continuação

\begin{tabular}{|c|c|c|c|c|}
\hline Variáveis & & & $\mathrm{FA}^{\mathrm{a}}$ & $\mathbf{F R}^{\mathrm{b}}$ \\
\hline \multirow{4}{*}{ Ingestão alimentar } & Alterou & & 45 & 68 \\
\hline & Não alterou & & 21 & 32 \\
\hline & Alteração da ingestão & Aumento & 24 & 53 \\
\hline & alimentar & Redução & 21 & 47 \\
\hline \multirow{4}{*}{$\begin{array}{l}\text { Prática de atividade } \\
\text { física }\end{array}$} & Alterou & & 47 & 71 \\
\hline & Não alterou & & 19 & 29 \\
\hline & Alteração da prática & Aumento & 7 & 15 \\
\hline & de atividade física & Redução & 40 & 85 \\
\hline
\end{tabular}

Os valores médios do peso e índice de massa corporal, bem como a variação de aumento e redução do peso, são apresentados na Tabela 2.

Tabela 2 - Variação e valores médios de peso corporal e IMC das mulheres. São Paulo, 2000-2008.

\section{Variáveis}

Peso corporal $(\mathbf{k g})$

IMC $\left(\mathrm{kg} / \mathrm{m}^{2}\right)$
Inicial

Final

Inicial

Final

\section{Valores médios}

65

67

25,43

26,08

2,9

$-1,4$ 
A maioria da mulheres classificadas como baixo peso, peso adequado, risco para obesidade e obesidade, apresentaram aumento de peso corporal (Tabela 3).

Tabela 3 - Distribuição das mulheres, segundo alteração de peso corporal, de acordo com classificação pelo IMC inicial. São Paulo, 2000-2008.

\section{Alteração de peso corporal (kg)}

\section{Classificação pelo \\ IMC $\left(\mathbf{k g} / \mathrm{m}^{2}\right)$}

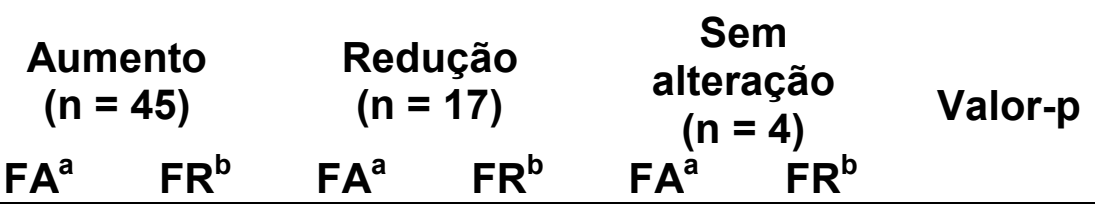

Baixo peso

$$
(n=1)
$$

Peso adequado

$(n=38)$

$23 \quad 35$

12

18

3

5

Risco de obesidade ( $n=18$ )

20

5

8

0

0

Obesidade

$(\mathrm{n}=9)$

812

0

0

1

1

Nota: ${ }^{a}$ : frequência absoluta; ${ }^{\text {: }}$ : frequência relativa.

${ }^{1}$ Teste $\chi^{2}$. 


\subsection{ALTERAÇÃO DE PESO E DE ÍNDICE DE MASSA CORPORAL}

\subsubsection{Quimioterapia Adjuvante}

Protocolos quimioterápicos adjuvantes, contendo antraciclinas, foram indicados para $74 \%$ das mulheres (Tabela 1 ).

Independente do tipo de protocolo, a maioria (48 e $20 \%$ com antraciclina e sem antraciclina, respectivamente) das mulheres apresentou aumento de peso corporal, sendo observada maior proporção naquelas submetidas a protocolos com antraciclina, embora a diferença não tenha sido estatisticamente significante (Tabela 4).

Tabela 4 - Distribuição das mulheres, de acordo com alteração de peso corporal e protocolos quimioterápicos adjuvantes. São Paulo, 2000-2008.

\footnotetext{
Protocolos

Alteração de peso corporal (kg) quimioterápicos adjuvantes Aumento Redução Sem alteração $(n=45)$ $(n=17)$ $F A^{\mathrm{a}}$ $F^{b} \quad F A^{a}$ $F^{a} \quad F^{a}$ $F A^{\mathrm{a}} \quad \mathrm{FR}^{\mathrm{b}}$

Valor-p

$\begin{array}{ccccccc}\begin{array}{c}\text { Com antraciclina } \\ (\mathbf{n}=\mathbf{4 9})\end{array} & 32 & 48 & 13 & 20 & 4 & 6 \\ \begin{array}{c}\text { Sem antraciclina } \\ (\mathbf{n}=17)\end{array} & 13 & 20 & 4 & 6 & 0 & 0\end{array}$

Nota: ${ }^{a}$ : frequência absoluta; ${ }^{b}$ : frequência relativa.

${ }^{1}$ Teste $\chi^{2}$.

O valor médio de aumento de peso corporal, entretanto, foi maior nas mulheres submetidas a protocolos sem antraciclina (Tabela 5), apesar da diferença não ter sido estatisticamente significante.
} 
Tabela 5 - Variação do peso corporal, segundo protocolos quimioterápicos adjuvantes. São Paulo, 2000-2008.

\begin{tabular}{ccc}
\hline $\begin{array}{c}\text { Protocolos quimioterápicos } \\
\text { adjuvantes }\end{array}$ & $\begin{array}{c}\text { Variação de peso corporal (kg) } \\
\text { Média (desvio-padrão) } \\
\text { (IC 95\%) }\end{array}$ & Valor-p \\
\hline Com antraciclina & $1,49(2,06)$ & \\
& $(0,90-2,09)$ & $0,40^{2}$ \\
Sem antraciclina & $2,11(3,87)$ & \\
& $(0,13-4,11)$ & \\
\hline
\end{tabular}

${ }^{2}$ Teste t-Student para amostras independentes.

Em relação ao IMC, $47 \%$ das mulheres que estavam com peso adequado e $27 \%$, com excesso de peso (16\%, risco para obesidade e $11 \%$, obesidade), foram submetidas a protocolos com antraciclina, no início do tratamento, enquanto $11 \%$ daquelas que estavam com peso adequado e $14 \%$ com excesso de peso ( $11 \%$, risco para obesidade e $3 \%$, obesidade), a protocolo sem antraciclina (Tabela 6 ). 
Tabela 6 - Distribuição das mulheres, segundo classificação do IMC inicial e protocolos quimioterápicos. São Paulo, 2000-2008.

\begin{tabular}{|c|c|c|c|c|c|c|c|c|c|}
\hline \multirow{4}{*}{$\begin{array}{l}\text { Protocolos } \\
\text { quimioterápicos }\end{array}$} & \multicolumn{9}{|c|}{ Classificação pelo IMC $\left(\mathrm{kg} / \mathrm{m}^{2}\right)$} \\
\hline & \multirow{2}{*}{\multicolumn{2}{|c|}{ Baixo peso }} & \multirow{2}{*}{\multicolumn{2}{|c|}{$\begin{array}{c}\text { Peso } \\
\text { adequado }\end{array}$}} & \multicolumn{4}{|c|}{ Excesso de peso } & \multirow{3}{*}{ Valor-p } \\
\hline & & & & & & de & Obe & dade & \\
\hline & $F^{a}$ & $\mathbf{F} \mathbf{R}^{\mathbf{b}}$ & $\mathrm{FA}^{\mathrm{a}}$ & $\mathbf{F R}^{\mathrm{b}}$ & $\mathrm{FA}^{\mathrm{a}}$ & $\mathbf{F R}^{\mathrm{b}}$ & $F^{a}$ & $\mathbf{F R}^{\mathbf{b}}$ & \\
\hline $\begin{array}{l}\text { Com antraciclina } \\
\qquad(n=49)\end{array}$ & 0 & 0 & 31 & 47 & 11 & 16 & 7 & 11 & \multirow[b]{2}{*}{$0,13^{1}$} \\
\hline $\begin{array}{l}\text { Sem antraciclina } \\
\qquad(n=17)\end{array}$ & 1 & 1 & 7 & 11 & 7 & 11 & 2 & 3 & \\
\hline \multicolumn{10}{|c|}{$\begin{array}{l}\text { Nota: }{ }^{a} \text { : frequência absoluta; }{ }^{b} \text { : frequência relativa. } \\
{ }^{1} \text { Teste } \chi^{2} \text {. }\end{array}$} \\
\hline $\begin{array}{r}\text { Quant } \\
\text { submetidas a } \\
\text { A maic } \\
\text { quimioterapia } \\
\text { corporal se } \\
\text { entretanto, q } \\
\text { apresentou } r \\
\text { ciclos (Tabela }\end{array}$ & $\begin{array}{l}\text { o ao } \\
\text { a seis, } \\
\text { oria c } \\
\text { ( } 56 \\
\text { ndo } \\
\text { que a } \\
\text { reduça } \\
\text { a } 7 \text { ). }\end{array}$ & $\begin{array}{l}\text { úmero } \\
\text { ndepen } \\
\text { s mulh } \\
8 \%, \\
\text { dife } \\
\text { maioria } \\
\text { de pe }\end{array}$ & $\begin{array}{l}\text { le cicl } \\
\text { dente } \\
\text { eres } \\
\text { espe } \\
\text { ença } \\
(9 \%)\end{array}$ & $\begin{array}{l}\text { de qu } \\
\text { ente dc } \\
\text { e se } s \\
\text { vamen } \\
\text { estatist } \\
\text { das } m \\
\text { oral, } p\end{array}$ & $\begin{array}{l}\text { niotera } \\
\text { orotocc } \\
\text { bmete } \\
\text { ), apr } \\
\text { ament } \\
\text { heres }\end{array}$ & $\begin{array}{l}\text { a, } 79 \% \\
\text { utiliza } \\
\text { n a se } \\
\text { entara } \\
\text { signi } \\
\text { ubmet }\end{array}$ & $\begin{array}{l}\text { das mb } \\
\text { (Tabe } \\
\text { ou m } \\
\text { aume } \\
\text { ante. } \\
\text { a a }\end{array}$ & $\begin{array}{l}\text { heres } \\
\text { 1). } \\
\text { is ciclo } \\
\text { to de } \\
\text { Dbserv } \\
\text { atro ci } \\
\text { nos o }\end{array}$ & $\begin{array}{l}\text { de } \\
\text { so } \\
\text { se, } \\
\text { os, } \\
\text { ros }\end{array}$ \\
\hline
\end{tabular}


Tabela 7 - Distribuição das mulheres, segundo alteração de peso corporal, de acordo com número de ciclos de quimioterapia adjuvante. São Paulo, 20002008.

\begin{tabular}{|c|c|c|c|c|c|c|c|}
\hline \multirow{3}{*}{$\begin{array}{l}\text { Número de } \\
\text { ciclos de } \\
\text { quimioterapia }\end{array}$} & \multicolumn{7}{|c|}{ Alteração de peso corporal (kg) } \\
\hline & \multicolumn{2}{|c|}{$\begin{array}{l}\text { Aumento } \\
(n=45)\end{array}$} & \multicolumn{2}{|c|}{$\begin{array}{l}\text { Redução } \\
(n=17)\end{array}$} & \multicolumn{2}{|c|}{$\begin{array}{c}\text { Sem alteração } \\
(n=4)\end{array}$} & \multirow[t]{2}{*}{ Valor-p } \\
\hline & $F A^{a}$ & $\mathbf{F R}^{\mathbf{b}}$ & $F A^{a}$ & $\mathbf{F R}^{\mathbf{b}}$ & $\mathrm{FA}^{\mathrm{a}}$ & $\mathbf{F R}^{\mathbf{b}}$ & \\
\hline $\begin{array}{l}4 \text { ciclos } \\
(n=9)\end{array}$ & 3 & 4 & 6 & 9 & 0 & 0 & \\
\hline $\begin{array}{l}6 \text { ciclos } \\
(n=52)\end{array}$ & 37 & 56 & 11 & 17 & 4 & 6 & $\mathbf{0 , 0 2} \mathbf{*}^{1}$ \\
\hline $\begin{array}{l}\text { Mais de } 6 \text { ciclos } \\
\quad(n=5)\end{array}$ & 5 & 8 & 0 & 0 & 0 & 0 & \\
\hline \multicolumn{8}{|c|}{$\begin{array}{l}\text { Nota: }{ }^{a} \text { : frequência absoluta; }{ }^{b} \text { :frequência relativa. } \\
\text { *Valor-p estatisticamente significativo; }{ }^{1} \text { Teste } \chi^{2} \text {. }\end{array}$} \\
\hline \multicolumn{8}{|c|}{$\begin{array}{l}\text { O valor médio de aumento de peso corporal foi maior nas mulheres } \\
\text { submetidas a } 6 \text { ou mais ciclos de quimioterapia (Tabela 8), apesar da diferença } \\
\text { não ter sido estatisticamente significante. }\end{array}$} \\
\hline \multicolumn{8}{|c|}{$\begin{array}{l}\text { Tabela } 8 \text { - Variação do peso corporal, de acordo com o número de ciclos de } \\
\text { quimioterapia adjuvante. São Paulo, } 2000-2008 \text {. }\end{array}$} \\
\hline \multicolumn{3}{|c|}{$\begin{array}{l}\text { Número de ciclos de } \\
\text { quimioterapia }\end{array}$} & \multicolumn{4}{|c|}{$\begin{array}{l}\text { Variação de peso corporal (kg) } \\
\text { Média (desvio-padrão) }\end{array}$} & Valor-p \\
\hline \multicolumn{3}{|c|}{4 ciclos } & \multicolumn{4}{|c|}{$0,07(2,0)$} & \\
\hline \multicolumn{3}{|c|}{6 ciclos } & \multicolumn{4}{|c|}{$1,75(2,6)$} & $0,055^{3}$ \\
\hline \multicolumn{3}{|c|}{ Mais de 6 ciclos } & \multicolumn{4}{|c|}{$3,48(1,3)$} & \\
\hline
\end{tabular}


Quanto ao IMC, a maioria (12 e 44\%) das mulheres que estava com peso adequado, no início da quimioterapia, se submeteu a quatro e seis ciclos, respectivamente, enquanto $33 \%$ (21\%, risco para obesidade e $12 \%$, obesidade) e $6 \%$ ( $5 \%$, risco para obesidade e $1 \%$, obesidade) daquelas que estavam com excesso de peso, a seis ou mais ciclos (Tabela 9).

Tabela 9 - Distribuição das mulheres, segundo classificação do IMC inicial e número de ciclos de quimioterapia. São Paulo, 2000-2008.

\begin{tabular}{|c|c|c|c|c|c|c|c|c|c|}
\hline \multirow{4}{*}{$\begin{array}{l}\text { Número de } \\
\text { ciclos de } \\
\text { quimioterapia }\end{array}$} & \multicolumn{9}{|c|}{ Classificação pelo IMC $\left(\mathrm{kg} / \mathrm{m}^{2}\right)$} \\
\hline & \multirow{2}{*}{\multicolumn{2}{|c|}{ Baixo peso }} & \multirow{2}{*}{\multicolumn{2}{|c|}{$\begin{array}{l}\text { Peso } \\
\text { adequado }\end{array}$}} & \multicolumn{4}{|c|}{ Excesso de peso } & \multirow[t]{3}{*}{ Valor-p } \\
\hline & & & & & \multicolumn{2}{|c|}{$\begin{array}{l}\text { Risco de } \\
\text { obesidade }\end{array}$} & \multicolumn{2}{|c|}{ Obesidade } & \\
\hline & $F^{a}$ & $\mathbf{F R}^{\mathbf{b}}$ & $\mathrm{FA}^{\mathrm{a}}$ & $\mathbf{F R}^{\mathbf{b}}$ & $\mathrm{FA}^{\mathrm{a}}$ & $\mathbf{F R}^{\mathbf{b}}$ & FA $^{a}$ & $\mathbf{F R}^{\mathbf{b}}$ & \\
\hline $\begin{array}{l}4 \text { ciclos } \\
(n=9)\end{array}$ & 0 & 0 & 8 & 12 & 1 & 1 & 0 & 0 & \\
\hline $\begin{array}{l}6 \text { ciclos } \\
(n=52)\end{array}$ & 1 & 1 & 29 & 44 & 14 & 21 & 8 & 12 & $0,23^{1}$ \\
\hline $\begin{array}{l}\text { Mais de } 6 \text { ciclos } \\
\qquad(n=5)\end{array}$ & 0 & 0 & 1 & 1 & 3 & 5 & 1 & 1 & \\
\hline \multicolumn{10}{|c|}{$\begin{array}{l}\text { Nota: }^{\text {a: }} \text { frequência absoluta; }{ }^{\text {: }} \text { frequência relativa. } \\
{ }^{1} \text { Teste } \chi^{2} \text {. }\end{array}$} \\
\hline \multicolumn{10}{|c|}{ 4.2.2 Fase do Climatério } \\
\hline \multicolumn{10}{|c|}{ A maioria (44 e $24 \%$, na pré e na pós-menopausa, respectivamente) das } \\
\hline
\end{tabular}


Tabela 10 - Distribuição das mulheres, de acordo com alteração de peso corporal e fase do climatério. São Paulo, 2000-2008.

\begin{tabular}{|c|c|c|c|c|c|c|c|}
\hline \multirow{3}{*}{ Fase do climatério } & \multicolumn{6}{|c|}{ Alteração de peso corporal (kg) } & \multirow{3}{*}{ Valor-p } \\
\hline & \multicolumn{2}{|c|}{$\begin{array}{c}\text { Aumento } \\
(\mathrm{n}=45)\end{array}$} & \multicolumn{2}{|c|}{$\begin{array}{c}\text { Redução } \\
(n=17)\end{array}$} & \multicolumn{2}{|c|}{$\begin{array}{c}\text { Sem alteração } \\
(n=4)\end{array}$} & \\
\hline & $F^{a}$ & $\mathrm{FR}^{\mathrm{b}}$ & $F A^{a}$ & $\mathrm{FR}^{\mathbf{b}}$ & $F A^{a}$ & $\mathrm{FR}^{\mathrm{b}}$ & \\
\hline $\begin{array}{l}\text { Pré-menopausa } \\
\qquad(n=43)\end{array}$ & 29 & 44 & 10 & 15 & 4 & 6 & \multirow[b]{2}{*}{$0,29^{1}$} \\
\hline $\begin{array}{l}\text { Pós-menopausa } \\
\quad(n=23)\end{array}$ & 16 & 24 & 7 & 11 & 0 & 0 & \\
\hline \multicolumn{8}{|c|}{ Nota: ${ }^{a}$ : frequência absoluta; ${ }^{b}$ : frequência relativa. } \\
\hline \multicolumn{8}{|c|}{$\begin{array}{l}\text { O valor médio de aumento do peso corporal foi similar em todas as } \\
\text { mulheres, pré e pós-menopausadas, sendo maior nessas últimas, mas sem } \\
\text { diferença estatisticamente significante (Tabela 11). As mulheres pós- } \\
\text { menopausadas com idade inferior a } 60 \text { anos, entretanto, apresentaram valor } \\
\text { médio de aumento de peso de } 2,4 \mathrm{~kg} \text {, enquanto aquelas com idade igual ou } \\
\text { maior que } 60 \text { anos, } 0,6 \mathrm{~kg} \text {, mostrando que, talvez, a idade interfira mais que a } \\
\text { fase do climatério no aumento de peso corporal em mulheres durante a } \\
\text { quimioterapia. }\end{array}$} \\
\hline
\end{tabular}


Tabela 11 - Variação do peso corporal, de acordo com a fase do climatério e grupo etário. São Paulo, 2000-2008.

\begin{tabular}{ccc}
\hline Fase do climatério & $\begin{array}{c}\text { Variação de peso corporal (kg) } \\
\text { Média (desvio-padrão) } \\
\text { (IC 95\%) }\end{array}$ & Valor-p \\
\hline Pré-menopausa & $1,59(2,14)$ & \\
Pós-menopausa & $(0,94-2,25)$ & $0,80^{2}$ \\
$-<60$ anos & $1,77(2,63)$ & \\
$-\geq 60$ anos & $(0,29-3,25)$ & $0,22^{2}$ \\
& $2,41(0,88)$ & \\
\hline
\end{tabular}

${ }^{2}$ Teste t-Student para amostras independentes.

Em relação ao $\mathrm{IMC}, 44 \%$ das mulheres que estavam com peso adequado e $22 \%$, com excesso de peso (14\%, risco para obesidade e $8 \%$, obesidade), estavam na pré-menopausa, enquanto $14 \%$ daquelas que estavam com peso adequado e $19 \%$ com excesso de peso (14\%, risco para obesidade e $5 \%$, obesidade), na pós-menopausa (Tabela 12 ). 
Tabela 12 - Distribuição das mulheres, segundo classificação do IMC inicial e fase do climatério. São Paulo, 2000-2008.

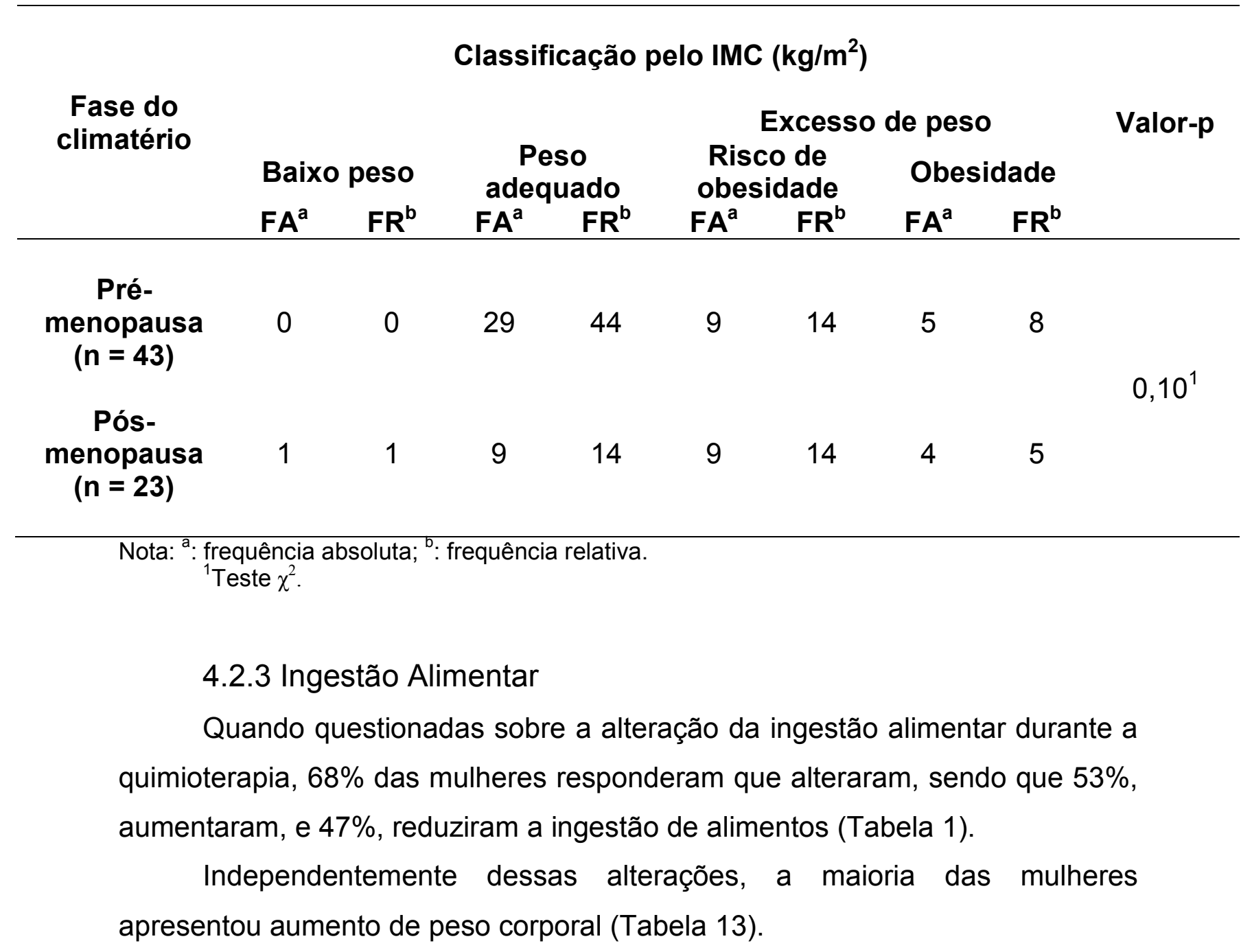


Tabela 13 - Distribuição das mulheres, de acordo com alteração de peso corporal e da ingestão alimentar. São Paulo, 2000-2008.

\begin{tabular}{|c|c|c|c|c|c|c|c|c|}
\hline \multirow{3}{*}{\multicolumn{2}{|c|}{ Ingestão alimentar }} & \multicolumn{6}{|c|}{ Alteração de peso corporal (kg) } & \multirow{3}{*}{ Valor-p } \\
\hline & & \multicolumn{2}{|c|}{$\begin{array}{c}\text { Aumento } \\
(n=45)\end{array}$} & \multicolumn{2}{|c|}{$\begin{array}{c}\text { Redução } \\
(n=17)\end{array}$} & \multicolumn{2}{|c|}{$\begin{array}{l}\text { Sem } \\
\text { alteração } \\
(n=4)\end{array}$} & \\
\hline & & $F^{a}$ & $\mathbf{F R}^{\mathbf{b}}$ & $F A^{a}$ & $\mathbf{F R}^{\mathbf{b}}$ & $F A^{a}$ & $\mathbf{F R}^{\mathbf{b}}$ & \\
\hline \multirow{2}{*}{\multicolumn{2}{|c|}{ Alterou $(n=45)$}} & 30 & 45 & 11 & 17 & 4 & 6 & \multirow{3}{*}{$0,37^{1}$} \\
\hline & & & & & & & & \\
\hline Não alterou & $(n=21)$ & 15 & 23 & 6 & 9 & 0 & 0 & \\
\hline \multirow{2}{*}{$\begin{array}{l}\text { Alteração da } \\
\text { ingestão } \\
\text { alimentar }\end{array}$} & $\begin{array}{c}\text { Aumento } \\
(\mathrm{n}=24)\end{array}$ & 17 & 38 & 5 & 11 & 2 & 4 & \multirow{2}{*}{$0,66^{1}$} \\
\hline & $\begin{array}{c}\text { Redução } \\
(n=21)\end{array}$ & 13 & 29 & 6 & 13 & 2 & 4 & \\
\hline
\end{tabular}

Nota: ${ }^{a}:$ frequência absoluta; ${ }^{b}$ : frequência relativa.
${ }^{1}$ Teste $\chi^{2}$.

Com relação à variação do peso corporal, constatou-se maior valor médio de aumento de peso, nas mulheres que alteraram a ingestão alimentar, sendo maior naquelas que referiram aumento da mesma, embora sem diferença estatisticamente significante (Tabela 14). 
Tabela 14 - Variação do peso corporal, de acordo com a ingestão alimentar. São Paulo, 2000-2008.

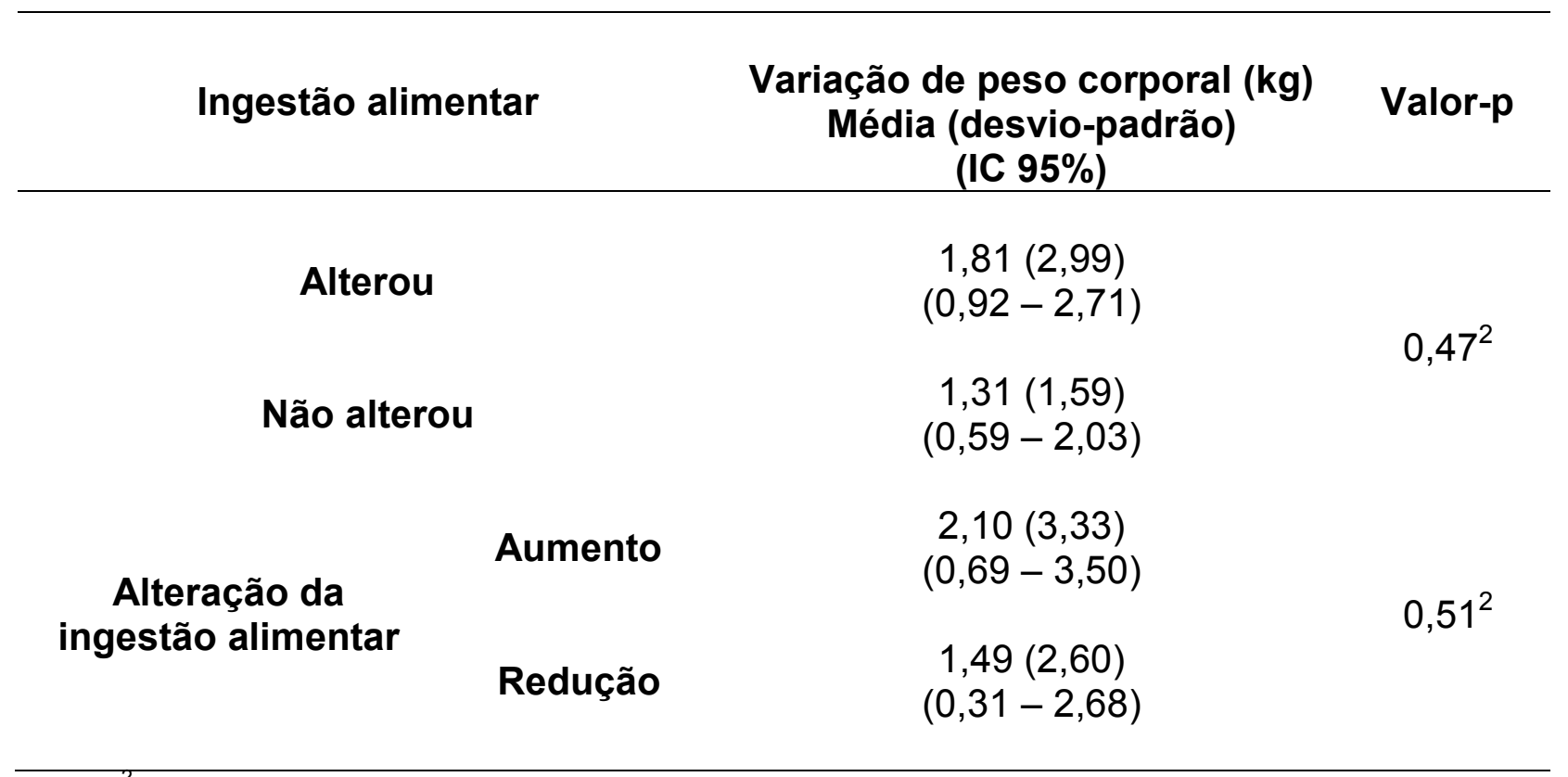

${ }^{2}$ Teste t-Student para amostras independentes.

Em relação ao IMC, 41\% das mulheres que estavam com peso adequado e $27 \%$, com excesso de peso (18\%, risco para obesidade e $9 \%$, obesidade), referiram alteração da ingestão alimentar, enquanto $17 \%$ daquelas que estavam com peso adequado e $14 \%$ com excesso de peso $(9 \%$, risco para obesidade e 5\%, obesidade), não referiram alteração (Tabela 15).

Das mulheres que alteraram a ingestão alimentar, independente de aumento ou redução da mesma, a maioria (33\% das que aumentaram e $27 \%$ das que reduziram) iniciou a quimioterapia com peso adequado e $20 \%$, com excesso de peso (risco para obesidade ou obesidade) (Tabela 15). 
Tabela 15 - Distribuição das mulheres, segundo classificação do IMC inicial e ingestão alimentar. São Paulo, 2000-2008.

\begin{tabular}{|c|c|c|c|c|c|c|c|c|c|c|}
\hline \multicolumn{10}{|c|}{ Classificação pelo IMC $\left(\mathrm{kg} / \mathrm{m}^{2}\right)$} & \multirow{4}{*}{ Valor-p } \\
\hline \multirow{3}{*}{\multicolumn{2}{|c|}{ Ingestão alimentar }} & \multirow{2}{*}{\multicolumn{2}{|c|}{$\begin{array}{l}\text { Baixo } \\
\text { peso }\end{array}$}} & \multirow{2}{*}{\multicolumn{2}{|c|}{$\begin{array}{c}\text { Peso } \\
\text { adequado }\end{array}$}} & \multicolumn{4}{|c|}{ Excesso de peso } & \\
\hline & & & & & & \multicolumn{2}{|c|}{$\begin{array}{l}\text { Risco de } \\
\text { obesidade }\end{array}$} & \multicolumn{2}{|c|}{ Obesidade } & \\
\hline & & & & $\mathrm{FA}^{\mathrm{a}}$ & $\mathbf{F R}^{\mathbf{b}}$ & $\mathrm{FA}^{\mathrm{a}}$ & $\mathbf{F R}^{\mathbf{b}}$ & $F^{a}$ & $\mathbf{F R}^{\mathbf{b}}$ & \\
\hline \multirow{4}{*}{$\begin{array}{l}\text { Alteração da } \\
\text { ingestão } \\
\text { alimentar }\end{array}$} & $=45)$ & 0 & 0 & 27 & 41 & 12 & 18 & 6 & 9 & \multirow{2}{*}{$0,51^{1}$} \\
\hline & $(n=21)$ & 1 & 1 & 11 & 17 & 6 & 9 & 3 & 5 & \\
\hline & $\begin{array}{l}\text { Aumento } \\
(n=24)\end{array}$ & 0 & 0 & 15 & 33 & 5 & 11 & 4 & 9 & \multirow{2}{*}{$0,57^{1}$} \\
\hline & $\begin{array}{c}\text { Redução } \\
(n=21)\end{array}$ & 0 & 0 & 12 & 27 & 7 & 16 & 2 & 4 & \\
\hline
\end{tabular}

Nota: ${ }^{a}$ : frequência absoluta; ${ }^{b}$ : frequência relativa. ${ }^{1}$ Teste $\chi^{2}$.

\subsubsection{Prática de Atividade Física}

A maioria das mulheres $(71 \%)$ referiu alteração da prática de atividade física, sendo que $85 \%$ reduziram e $15 \%$, aumentaram (Tabela 1 ).

Houve maior proporção de mulheres com aumento de peso entre aquelas que alteraram a prática de atividade física (55\%) e essa diferença foi estatisticamente significativa $(p=0,04)$ (Tabela 16).

Ao analisar a proporção de mulheres com alteração de peso corporal considerando as que alteraram a prática de atividade física, verifica-se que a maioria (13 e 64\% das que aumentaram ou reduziram, respectivamente) também apresentou aumento de peso corporal, mas sem diferença estatisticamente significante (Tabela 16). 
Tabela 16 - Distribuição das mulheres de acordo com alteração de peso corporal e prática de atividade física. São Paulo, 2000-2008.

\begin{tabular}{|c|c|c|c|c|c|c|c|c|}
\hline \multicolumn{8}{|c|}{ Alteração de peso corporal (kg) } & \multirow{3}{*}{ Valor-p } \\
\hline \multirow{2}{*}{\multicolumn{2}{|c|}{$\begin{array}{l}\text { Prática de atividade } \\
\text { física }\end{array}$}} & \multicolumn{2}{|c|}{$\begin{array}{l}\text { Aumento } \\
(n=45)\end{array}$} & \multicolumn{2}{|c|}{$\begin{array}{l}\text { Redução } \\
(n=17)\end{array}$} & \multicolumn{2}{|c|}{$\begin{array}{c}\text { Sem } \\
\text { alteração } \\
(n=4)\end{array}$} & \\
\hline & & $F^{a}$ & $\mathbf{F R}^{\mathbf{b}}$ & $F^{a}$ & $\mathbf{F R}^{\mathbf{b}}$ & $F^{a}$ & $\mathbf{F R}^{\mathbf{b}}$ & \\
\hline \multirow{2}{*}{\multicolumn{2}{|c|}{ Alterou $(n=47)$}} & 36 & 55 & 8 & 12 & 3 & 4 & \multirow{3}{*}{$0,04^{* 1}$} \\
\hline & & & & & & & & \\
\hline \multicolumn{2}{|c|}{ Não alterou (n = 19) } & 9 & 14 & 9 & 14 & 1 & 1 & \\
\hline \multirow{2}{*}{$\begin{array}{c}\text { Alteração } \\
\text { da prática } \\
\text { de } \\
\text { atividade } \\
\text { física }\end{array}$} & $\begin{array}{l}\text { Aumento } \\
(n=7)\end{array}$ & 6 & 13 & 1 & 2 & 0 & 0 & \multirow[b]{2}{*}{$0,12^{1}$} \\
\hline & $\begin{array}{c}\text { Redução } \\
(n=40)\end{array}$ & 30 & 64 & 7 & 15 & 3 & 6 & \\
\hline \multicolumn{9}{|c|}{$\begin{aligned} & \text { Nota: }{ }^{1}: \text { frequência absoluta; }{ }^{2} \text { : frequência relativa. } \\
& { }^{*} \text { Valor-p estatisticamente significativo; }{ }^{1} \text { Teste } \chi^{2} .\end{aligned}$} \\
\hline alterara & $\begin{array}{c}\text { valor médio } \\
\text { a prática } \\
\text { te (Tabela }\end{array}$ & aum & de p & $\begin{array}{l}\text { corp } \\
\text { mas }\end{array}$ & m dif & ença & $\begin{array}{l}\text { mulhe } \\
\text { tatistic }\end{array}$ & $\begin{array}{l}\text { que } \\
\text { mente }\end{array}$ \\
\hline
\end{tabular}


Tabela 17 - Variação do peso corporal, de acordo com a prática de atividade física atividade física. São Paulo, 2000-2008.

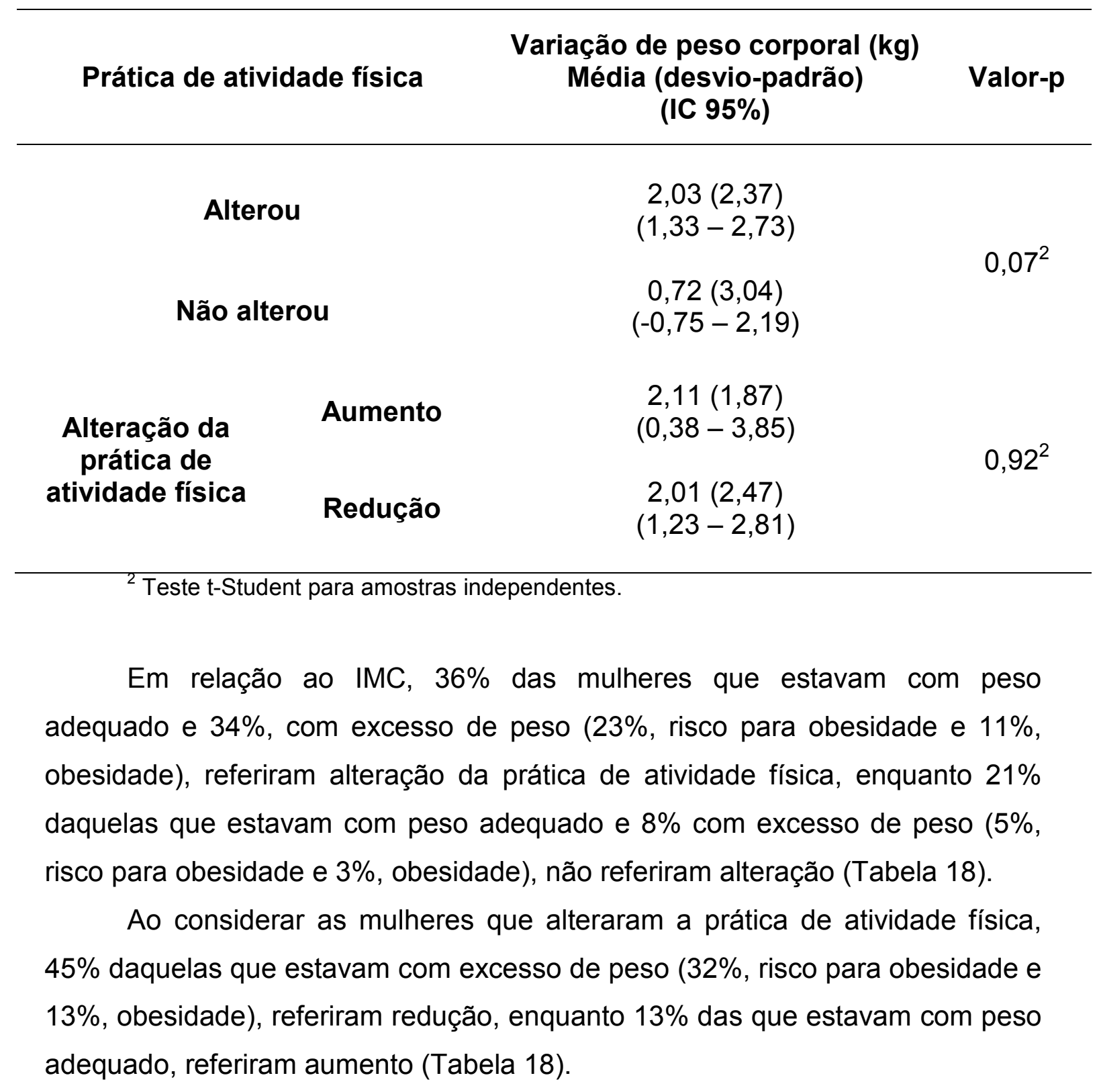


Tabela 18 - Distribuição das mulheres, segundo classificação do IMC inicial e prática de atividade física. São Paulo, 2000-2008.

\begin{tabular}{|c|c|c|c|c|c|c|c|c|c|c|}
\hline \multicolumn{11}{|c|}{ Classificação pelo IMC (kg/m²) } \\
\hline \multirow{3}{*}{\multicolumn{2}{|c|}{$\begin{array}{l}\text { Prática de atividade } \\
\text { física }\end{array}$}} & \multirow{2}{*}{\multicolumn{2}{|c|}{$\begin{array}{l}\text { Baixo } \\
\text { peso }\end{array}$}} & \multirow{2}{*}{\multicolumn{2}{|c|}{$\begin{array}{c}\text { Peso } \\
\text { adequado }\end{array}$}} & \multicolumn{4}{|c|}{ Excesso de peso } & \multirow[t]{3}{*}{ Valor-p } \\
\hline & & & & & & $\begin{array}{l}\text { Risc } \\
\text { obes }\end{array}$ & & Obes & idade & \\
\hline & & & $\mathrm{FR}^{\mathrm{b}}$ & $F A^{a}$ & $\mathbf{F R}^{\mathrm{b}}$ & $\mathrm{FA}^{\mathrm{a}}$ & $F^{b}$ & $\mathrm{FA}^{\mathrm{a}}$ & $\mathbf{F R}^{\mathbf{b}}$ & \\
\hline \multicolumn{2}{|c|}{ Alterou $(n=47)$} & 1 & 1 & 24 & 36 & 15 & 23 & 7 & 11 & \multirow{2}{*}{$0,38^{1}$} \\
\hline \multicolumn{2}{|c|}{ Não alterou ( $n=19$ ) } & 0 & 0 & 14 & 21 & 3 & 5 & 2 & 3 & \\
\hline \multirow{2}{*}{$\begin{array}{c}\text { Alteração } \\
\text { da prática } \\
\text { de } \\
\text { atividade } \\
\text { física }\end{array}$} & $\begin{array}{c}\text { Aumento } \\
(\mathrm{n}=7)\end{array}$ & 0 & 0 & 6 & 13 & 1 & 2 & 0 & 0 & \multirow{2}{*}{$0,25^{1}$} \\
\hline & $\begin{array}{c}\text { Redução } \\
(n=40)\end{array}$ & 1 & 1 & 18 & 38 & 15 & 32 & 6 & 13 & \\
\hline
\end{tabular}




\subsection{DISCUSSÃO}

O aumento do peso corporal é comum em mulheres, submetidas à quimioterapia adjuvante. Foi descrito, primeiramente, por DIXON et al. (1978) e citado por DEMARK-WAHNEFRIED et al. (2001), tendo sido motivo de investigação, apesar de não estar, diretamente, relacionado às náuseas, vômitos e mucosites, cuja presença é frequente, durante o tratamento.

A etiologia do aumento de peso corporal, durante a quimioterapia adjuvante, ainda não é totalmente conhecida, e, embora não tenha sido objeto deste trabalho, estudos sugerem que a depressão e a alteração da percepção da imagem corporal que, comumente, acontecem após a cirurgia da mama, podem ocasionar aumento do apetite, redução da atividade física e do gasto energético. Além disso, é associado aos medicamentos quimioterápicos, à quimioterapia oral e ao número de ciclos da quimioterapia (CAMORIANO et al., 1990; DEMARK-WAHNEFRIED et al., 1997; KUTYNEC et al., 1999; ROONEY e WALD, 2007).

No presente estudo, $68 \%$ das mulheres apresentaram aumento de peso corporal, com valor médio de variação de $2,9 \mathrm{~kg}$. Estes resultados são semelhantes aos de outros autores.

IRWIN et al. (2005) avaliaram 514 mulheres com câncer de mama e constataram que $68 \%$ apresentaram aumento de peso corporal, com média de $3,9 \mathrm{~kg}$.

DEMARK-WAHNEFRIED et al. (1993 e 1997) verificaram aumento de peso corporal em $50 \%$ a $96 \%$ das pacientes com câncer de mama, durante a quimioterapia adjuvante, sendo que a média variou de 2,0 a 6,0 kg, no primeiro ano, após o diagnóstico.

GOODWIN et al. (1999) estudaram 535 mulheres com câncer de mama e verificaram que a maioria ( $84 \%$ ) teve aumento de peso corporal, sendo que aquelas que foram submetidas à quimioterapia adjuvante, apresentaram aumento médio de $2,5 \mathrm{~kg}$. 
MAKARI-JUDSON et al. (2007) avaliaram 185 mulheres com câncer de mama e constataram aumento significativo de peso $(2,6 \mathrm{~kg}$; $\mathrm{p}<0,0001)$ naquelas que se submeteram à quimioterapia adjuvante.

COSTA et al. (2002) realizaram estudo de coorte retrospectiva, com 106 pacientes com câncer de mama, durante a quimioterapia e constataram aumento de peso corporal, estatisticamente significante $(p<0,00001)$, em $81 \%$ das mulheres, com quimioterapia adjuvante. $O$ aumento foi de $0,91 \%(d p=1,19)$ do peso corporal, por mês de tratamento.

Segundo revisão de CHLEBOWSKI et al. (2002), vários autores têm encontrado associação positiva entre excesso de peso (IMC $\geq 25 \mathrm{~kg} / \mathrm{m}^{2}$ ) e recidiva da doença, bem como com mortalidade por câncer de mama, e consequentemente, com redução do tempo de vida das mulheres com essa doença.

PIERCE et al. (2007) avaliaram 1490 mulheres com câncer de mama e constataram que o IMC foi relacionado com mortalidade $(p=0,06)$. As mulheres obesas (IMC $\geq 30 \mathrm{~kg} / \mathrm{m}^{2}$ ) apresentaram taxa de mortalidade mais elevada do que aquelas com peso adequado $(p=0,01)$.

Neste estudo, $57 \%(51 \%<60$ anos e $6 \% \geq 60$ anos $)$ das mulheres apresentaram peso adequado, quando iniciaram a quimioterapia e $41 \%$ excesso de peso, sendo $27 \%$ (24\% $<60$ anos e $3 \% \geq 60$ anos), com risco para obesidade, e $14 \%$ ( $12 \%<60$ anos e $2 \% \geq 60$ anos), com obesidade. Os valores médios de IMC, inicial e final, foram 25 e $26 \mathrm{~kg} / \mathrm{m}^{2}$, respectivamente .

O aumento de peso corporal ocorreu na maioria (35\%) das mulheres que apresentaram peso adequado, no início do tratamento, em $32 \%$ daquelas, com excesso de peso (20\%, com risco para obesidade, e $12 \%$, com obesidade) , e também na única mulher, que apresentou baixo peso.

Vários autores (DEMARK-WAHNEFRIED et al., 1997; ROCK et al., 1999; INGRAM e BROWN, 2004; HARVIE et al., 2004; MARAKI-JUDSON et al., 2007) referiram aumento do peso corporal, em mulheres, submetidas à quimioterapia, 
e sua associação com agentes quimioterápicos e tempo de duração do tratamento.

No presente estudo, maior proporção (48\%) de mulheres, que apresentaram aumento de peso corporal, foram aquelas submetidas ao protocolo com antraciclina, porém a variação do valor médio do peso corporal foi maior $(2,11 \mathrm{~kg})$ naquelas sem antraciclina, porém, sem diferença estatisticamente significante.

Estes resultados são semelhantes aos de outros autores (ROCK et al., 1999; INGRAM e BROWN, 2004; DEL RIO et al., 2002), que constataram maior valor médio de aumento de peso corporal nas mulheres submetidas a protocolo sem antraciclina, com diferença estatisticamente significante $(p \leq 0,001)$.

LANKESTER et al. (2002), entretanto, não encontraram diferença, estatisticamente significante, $(p=0,86)$ de aumento de peso em mulheres submetidas à quimioterapia com e sem antraciclinas (FEC e CMF, respectivamente), embora tenham apresentado aumento significativo ( $p<$ 0,001 ) de peso corporal (média de $3,68 \mathrm{~kg}$ ) durante o tratamento.

Com relação ao número de ciclos de quimioterapia, $79 \%$ das mulheres submeteram-se a seis, $14 \%$, a quatro e $7 \%$, a mais de seis ciclos.

Foi verificado aumento do peso corporal em $64 \%$ das mulheres submetidas a seis ou mais de seis ciclos ( $56 \%$ e $8 \%$, respectivamente), e $4 \%$ naquelas submetidas a quatro, sendo que esses resultados foram estatisticamente significantes $(p=0,02)$. A variação do valor médio de peso corporal foi maior nas mulheres submetidas a mais de seis ciclos $(3,48 \mathrm{~kg})$ e menor, nas submetidas a quatro ciclos $(0,07 \mathrm{~kg})$.

Esses resultados corroboram os de vários autores, que também verificaram maior aumento de peso em mulheres submetidas à maior número de ciclos, provavelmente devido ao maior tempo de contato com medicamentos (DEMARK-WAHNEFRIED et al., 1993 e 1997; CAMORIANO et al., 1990; ROCK et al., 1999; KUTYNEC et al., 1999). 
Independente do protocolo e número de ciclos de quimioterapia, vários autores (COSTA et al., 2002; DEMARK-WAHNEFRIED et al., 1993 e 1997; GOODWIN et al., 1999; LANKESTER et al., 2002; MARAKI-JUDSON et al., 2007) referem a fase do climatério (pré e pós-menopausa), como fator de risco para o aumento de peso corporal, em mulheres com câncer de mama, principalmente, na pré-menopausa.

No presente estudo, $44 \%$ das mulheres, que tiveram aumento de peso corporal, eram pré-menopausadas, e $24 \%$, pós-menopausadas. A variação média de peso corporal foi maior nas mulheres pós-menopausadas $(1,77 \mathrm{~kg})$, sendo que houve maior diferença quando se considerou grupo etário < ou $\geq 60$ anos. Neste caso, a variação média foi maior nas mulheres com idade inferior a 60 anos (2,4 e 0,6 kg, respectivamente), embora sem diferença, estatisticamente significante.

Estes resultados corroboram os de ROCK et al. (1999), que avaliaram 1116 mulheres com câncer de mama e não constataram diferença significante entre aumento de peso corporal e fase do climatério, mas verificaram que, as mulheres pós-menopausadas com idade inferior a 50 anos, apresentaram aumento de peso corporal maior que $10 \%(p=0,007)$, do que as pósmenopausadas com idade superior a 50 anos e as pré-menopausadas.

IRWIN et al. (2005), em estudo com 514 mulheres com câncer de mama, verificaram que as pós-menopausadas mais jovens (40 - 49 anos) apresentaram maior variação do valor médio de peso $(5,2 \mathrm{~kg})$, do que as idosas (60 anos e mais) $(0,5 \mathrm{~kg}, \mathrm{p}<0,05)$.

Entretanto, a maioria dos estudos (CAMORIANO et al., 1990; DEMARKWAHNEFRIED et al., 1997; MAKARI-JUDSON et al., 2007), mostram associação entre aumento de peso durante a quimioterapia e fase do climatério, principalmente, a pré-menopausa.

Segundo DEMARK-WAHNEFRIED et al. (1993), o aumento de peso corporal que acontece durante a quimioterapia, em mulheres com câncer de 
mama, está relacionado ao aumento da ingestão alimentar e/ou redução do gasto energético.

Entretanto, neste estudo, esses resultados não puderam ser confirmados, pois, não foi possível obter dados detalhados sobre a ingestão alimentar (quantidade, qualidade e/ou tipo de alimentos) e à prática de atividade física (frequência, intensidade, duração) por se tratar de estudo retrospectivo, com coleta de dados a partir dos prontuários, nos quais não constavam essas informações. Além disso, as respostas fornecidas por telefone podem ter sido prejudicadas, tendo em vista que muitas mulheres haviam terminado o tratamento há mais de um ano, com comprometimento da lembrança a respeito dessas variáveis.

Verificou-se maior proporção, não estatisticamente significante, de mulheres com aumento de peso corporal, tanto naquelas que referiram alteração da ingestão alimentar (45\%), quanto naquelas que referiram não haver alteração (23\%).

O estudo Women's Healthy Eating and Living (WHEL), realizado nos Estados Unidos, durante seis anos, abrangeu 3100 mulheres com câncer de mama, pré e pós-menopausadas, com o objetivo de verificar a influência da alimentação na recidiva da doença. As mulheres foram distribuídas em dois grupos: controle (receberam orientação alimentar de consumo de frutas, verduras e legumes, cinco vezes ao dia, no mínimo $20 \mathrm{~g}$ de fibras e $30 \%$ do valor calórico da alimentação, referente à ingestão de gordura) e de intervenção (ingestão de legumes e verduras, cinco a seis vezes ao dia, frutas, três vezes, $30 \mathrm{~g}$ de fibras e 15 a $20 \%$ do valor calórico da alimentação, referente à gordura).

No primeiro ano de acompanhamento, ROCK et al.(2001) constataram que $74 \%$ das 1010 mulheres estudadas (grupo controle e intervenção), não apresentaram alteração de peso corporal, 11\% apresentaram redução e 15\%, aumento, sem diferença, estatisticamente significante, entre alteração de peso corporal e ingestão alimentar. 
PIERCE et al. (2007), após seis anos do WHEL, verificaram que o grupo controle não apresentou alteração significativa da ingestão de frutas, legumes e verduras, mas apresentou $13 \%$ de aumento na ingestão de alimentos, com alto teor de gordura. O grupo intervenção, alterou, significativamente $(p<0,001)$, a alimentação. Não houve aumento de peso maior que $1 \mathrm{~kg}$ entre os grupos. Com relação à recidiva e mortalidade por câncer de mama, ambas foram similares nos dois grupos (em torno de $17 \%$ das mulheres apresentaram recidiva da doença e $10 \%$, morreram). Os autores, portanto, não constataram evidências de que a alimentação do grupo intervenção tenha prevenido a recidiva e mortalidade por esta doença.

De acordo com MALZYNER e CAMPONERO (2004), a alteração da ingestão alimentar pode estar relacionada aos efeitos colaterais da quimioterapia, como por exemplo, alteração do paladar, que está associada aos agentes quimioterápicos. Os sintomas mais, frequentemente, relatados são o gosto metálico persistente e alterações na percepção da salinidade dos alimentos. Muitas mulheres, portanto, tendem a preferir alimentos doces e rejeitar carne vermelha, situação que pode conduzir ao desequilíbrio nutricional.

MAUNSELL et al. (2002) avaliaram alterações da ingestão alimentar em 250 mulheres com câncer de mama, um ano após o diagnóstico, e verificaram que, daquelas que referiram alteração (41\%), $77 \%$ reduziram a ingestão de carne vermelha, $72 \%$ aumentaram frutas, verduras e legumes e $66 \%$, aumentaram a ingestão de alimentos doces.

No presente estudo, apesar de não ter sido estatisticamente significante, as mulheres que referiram aumento da ingestão alimentar, apresentaram maior variação de peso corporal $(2,10 \mathrm{~kg})$, do que aquelas que referiram redução da ingestão alimentar (1,49 kg).

Em relação ao IMC, $27 \%$ das mulheres, que estavam com excesso de peso ( $18 \%$, com risco de obesidade e $9 \%$, com obesidade), referiram alteração da ingestão alimentar e, independentemente, do tipo de alteração (aumento ou 
redução), $20 \%$ estavam com excesso de peso ( $11 \%$, com risco de obesidade e $9 \%$, com obesidade).

Vários autores (BASTARRACHEA et al., 1994; CHLEBOWSKI et al., 2002 ; BERCLAZ et al., 2004; LOI et al., 2005 ; TAO et al., 2006) têm mostrado que as mulheres obesas (IMC $\geq 30 \mathrm{~kg} / \mathrm{m}^{2}$ ), bem como aquelas que apresentam aumento do peso corporal, após o diagnóstico, apresentam maior risco de recidiva da doença e menor tempo de vida.

Sendo assim, muitos pesquisadores têm estudado alimentação e risco de recidiva do câncer de mama e as conclusões, apesar de não serem consensuais, têm mostrado que a redução da ingestão de alimentos, com alto teor de gordura, e o aumento da ingestão de alimentos, com alto teor de fibras, vitaminas e minerais, contribuem para redução do peso corporal e, consequentemente, para redução do risco de recidiva da doença (HOLMES et al., 1999; THOMSON et al., 2005; McELIGOT et al., 2006; DOYLE et al., 2006; CHLEBOWSKI et al., 2006; ROONEY e WALD, 2007).

GOODWIN et al. (2003) analisaram a alteração da ingestão alimentar com tempo de vida, em 477 mulheres com câncer de mama, e constataram que alimentação adequada (ingestão de nutrientes, em quantidade e/ou qualidade recomendadas), esteve associada ao maior tempo de vida dessas mulheres.

KWAN et al. (2008) estudaram a associação entre a "alimentação adequada" (prudent pattern), e "inadequada" (western pattern), ou seja, que não atende, quantitativa e/ou qualitativamente, às recomendações dietéticas. Os autores constataram que as mulheres que se alimentaram adequadamente, apresentaram menor variação do aumento de peso $(1,6 \mathrm{~kg} ; \mathrm{p}=0,04)$ e eram mais ativas fisicamente $(p<0,0001)$, enquanto as que se alimentaram inadequadamente, apresentaram maior variação do valor médio de aumento de peso corporal $(2,7 \mathrm{~kg} ; \mathrm{p}=0,0002)$.

O Women's Intervention Nutrition Study (WINS), realizado nos Estados Unidos, envolveu 2400 mulheres, com câncer de mama, pós-menopasaudas, 
randomizadas em grupo controle (receberam orientação alimentar, mas sem modificação da alimentação), e de intervenção (redução de $15 \%$ do valor calórico da alimentação, referente à ingestão de gordura). Os autores verificaram que, após, aproximadamente, cinco anos de acompanhamento, o grupo intervenção, apresentou risco $24 \%$ menor de recidiva, que o grupo controle $(O R=0,76 ;$ IC $95 \%=0,60$ a 0,98$)$, sugerindo que, a redução da ingestão de gordura, pode aumentar o tempo de vida das mulheres e reduzir o risco de recidiva da doença. Houve, entretanto, diferença, estatisticamente significante $(p=0,005)$, no peso corporal médio das mulheres do grupo intervenção, com redução de $2,7 \mathrm{~kg}$, que pode ter interferido nos resultados (CHLEBOWSKI et al., 2006).

Estudos de intervenção, com objetivo de avaliar o efeito da redução do peso corporal em mulheres com câncer de mama, foram sumarizados em revisão feita por CHLEBOWSKI et al. (2002). Esses autores concluíram que intervenções para redução do peso corporal devem ser implementadas nessas mulheres, ainda durante a quimioterapia.

LOPRINZI et al. (1996) avaliaram 107 mulheres com câncer de mama, no início da quimioterapia e constataram que o grupo intervenção, que recebeu orientação alimentar adequada, apresentou redução média de $3,5 \mathrm{~kg}$, após 6 meses de acompanhamento, enquanto o grupo controle, apresentou aumento médio de $2 \mathrm{~kg}$. Ressalta-se que, aproximadamente, dois terços das mulheres, estavam com peso adequado, no início desse estudo.

O aumento de peso corporal também pode ocorrer, devido à redução da prática de atividade física, durante o tratamento, tornando-se importante fator de risco para recidiva da doença e redução do tempo de vida, uma vez que tem sido associada com menores níveis de hormônios ovarianos circulantes (McTIERNAN et al., 2003; HOLICK et al., 2008; IRWIN et al., 2004; IRWIN, 2009). 
HOLMES et al. (2005) avaliaram 2987 mulheres com câncer de mama e verificaram que a prática de atividade física, após o diagnóstico, reduziu o risco de morte pela doença, sendo que as mulheres que caminhavam de 3 a 5 horas semanais, foram as mais beneficiadas.

No presente estudo, a maioria (55\%) das mulheres que apresentaram aumento de peso corporal, referiu alteração da prática de atividade física, com diferença, estatisticamente, significante $(p=0,04)$, sendo que $64 \%$ referiram redução.

A variação de peso corporal foi maior (média de 2,03 kg) naquelas que alteraram a prática de atividade física, embora sem diferença estatisticamente significante, sendo que a variação de peso corporal foi similar a das que aumentaram (média de 2,01 e 2,11 kg, respectivamente).

Estes resultados corroboram os de IRWIN et al. (2003), que avaliaram 812 mulheres com câncer de mama e constataram a redução do tempo de prática de atividade física em $11 \%$, equivalente a 2 horas por semana, desde o ano anterior ao diagnóstico até 1 ano após. As mulheres submetidas à quimioterapia apresentaram maior redução $(50 \%)$, do que aquelas que fizeram apenas cirurgia $(24 \%)$ ou radioterapia $(23 \%)(p<0,05)$.

KUMAR et al. (2004) avaliaram 198 mulheres com câncer de mama, submetidas a quimioterapia, e verificaram que $56 \%$ reduziram a prática de atividade física, sendo que aquelas que apresentaram aumento de peso maior que $2 \mathrm{~kg}$, reduziram, em média, 6,5 horas por semana.

Quanto ao IMC, $34 \%$ das mulheres (23\% com risco de obesidade e $11 \%$, com obesidade), que referiram alteração da prática de atividade física, estavam com excesso de peso. Das mulheres que referiram alteração, verificou-se excesso de peso, principalmente, naquelas que reduziram $(32 \%$ com risco de obesidade e $13 \%$ com obesidade).

Resultados semelhantes foram obtidos por HOLMES et al. (2005), que avaliaram 4484 mulheres com câncer de mama, durante a quimioterapia e 
constataram que as menos ativas fisicamente ( $<3$ MET-h/semana), eram aquelas com risco de obesidade e obesidade (IMC $\geq 25 \mathrm{~kg} / \mathrm{m}^{2}$ ).

SCHWARTZ (2000) avaliou 78 mulheres com câncer de mama, no início da quimioterapia, e constataram que aquelas que mantiveram a prática de atividade física, durante o tratamento, apresentaram menor variação média do aumento de peso corporal (1,8 kg), do que as que não praticaram $(5,4 \mathrm{~kg})$.

ROONEY e WALD (2007), em revisão da literatura, verificaram que muitos estudos têm mostrado efeitos benéficos da intervenção da prática de atividade física na manutenção do peso corporal adequado, aumento da força muscular, da flexibilidade, redução ou alívio de e de alguns efeitos colaterais da quimioterapia, como náusea, fadiga, dor, ansiedade e depressão e melhora da qualidade de vida.

PINTO e TRUNZO (2004) avaliaram 119 mulheres com câncer de mama e constataram que aquelas que praticavam atividade física regularmente, apresentaram mais vigor e menos fadiga, do que as sedentárias.

$O$ aumento de peso corporal, que acomete as mulheres com câncer de mama, principalmente as idosas e pós-menopausadas, ocorre devido ao aumento da gordura, e não, da massa muscular. Essa condição é conhecida como obesidade sarcopênica, que está associada a hipopituitarismo, doenças neuromusculares, sedentarismo e uso crônico de corticosteróides, sendo que a prática de atividade física, especialmente treinamentos de resistência nos membros inferiores, e a melhora da qualidade da alimentação, podem prevenir e/ou controlar esse tipo de obesidade (DEMARK-WAHNEFRIED et al., 2002).

A Sociedade Americana de Câncer (ACS) recomenda a ingestão diária, pelo menos 5 vezes ao dia, de frutas, verduras e legumes e prática de atividade física moderada, pelo menos, 150 minutos por semana (DOYLE et al., 2006).

BLANCHARD et al. (2008) avaliaram 2.885 mulheres americanas, com câncer de mama, e constataram que a maioria desconhecia as recomendações 
da ACS, para alimentação $(81,8 \%)$ e para atividade física $(62,9 \%)$, mostrando a importância de intervenções para mudança de estilo de vida dessas mulheres.

Os resultados deste estudo não podem ser extrapolados para outras mulheres com câncer de mama, pois trata-se de amostra de conveniência, com número pequeno de mulheres (66), atendidas em clínica particular, da cidade de São Paulo. Sendo assim, elas não representaram a população com câncer de mama dessa cidade, uma vez que a amostra não foi representativa, e ainda, o nível socioeconômico, provavelmente, foi fator influenciador dos resultados. Ainda é importante ressaltar que a coleta de dados baseou-se nos prontuários dessas mulheres, e abrangeu longo período (2000-2008), sendo complementada com contato telefônico, realizado recentemente, fato que pode ter comprometido a fidedignidade dos resultados, devido à possibilidade de dificuldades, relacionados à memória. A "recusa" elevada das mulheres que não participaram do estudo relacionou-se, provavelmente, ao fato das mesmas não terem tido disponibilidade ou interesse em devolver o Termo de Consentimento assinado, pelo correio, mesmo tendo sido enviado envelope selado, pago pela pesquisadora, e da boa receptividade durante contato telefônico.

\subsection{CONCLUSÕES}

Com base nos resultados obtidos, conclui-se que:

- o maior o número de ciclos e a prática de atividade física, foram estatisticamente significantes, com alteração do peso corporal; enquanto o protocolo quimioterápico, a fase do climatério (pré e pós-menopausa) e a ingestão alimentar não alteraram, significativamente, o peso corporal;

- não houve diferença, estatisticamente significante, entre os valores médios de IMC, no início da quimioterapia, e as outras variáveis do estudo. 


\subsection{REFERÊNCIAS}

Aapro MS. Adjuvant therapy of primary breast cancer: a review of key findings from the 7th international conference, St. Gallen, February, 2001. The Oncologist. 2001; 6: 376-85.

Barros ACSD, Barbosa EM, Gebrim LH, Anelli A, Figueira Filho A, Del Giglio A et al. Diagnóstico e tratamento do câncer de mama. Projeto Diretrizes. Associação Médica Brasileira e Conselho Federal de Medicina. 2001.

Bastarrachea J, Hortobagyi GN, Smith TL, Kau SWC, Buzdar AU. Obesity as an adverse prognostic factor for patients receiving adjuvant chemotherapy for breast cancer. Ann Intern Med. 1994; 120 (1): 18-25.

Berclaz G, Li S, Price KN, Coates AS, Castiglione-Gertsch M, Rudenstam C-M, et al. Body mass index as a prognostic feature in operable breast cancer: the International Breast Cancer Study Group experience. Annals of Oncology. 2004; 15: 875-84.

Blanchard CM, Courneya KS, Stein K. Cancer Survivors` adherence to lifestyle behavior recommendations and associations with health-related quality of life: results from the American Cancer Society's SCS-II. J Clin Oncol. 2008; 26: 2198-204.

Bonassa EMA. Conceitos gerais em quimioterapia antineoplásica. In: Bonassa EMA e Santana TR. Enfermagem em terapêutica oncológica. $3^{a}$ edição. São Paulo: Atheneu; 2005. p. 3-19.

Brasileiro Filho G, Pereira FEL, Guimarães RC. Distúrbios do crescimento e da diferenciação celular. In: Brasileiro Filho G. Bogliolo Patologia. $7^{a}$ ed. Rio de Janeiro: Guanabara Koogan; 2006. p. 175-236.

Camoriano JK, Loprinzi CL, Ingle JN, Therneau TM, Krook JE, Veeder MH. Weight change in women treated with adjuvant therapy or observed following mastectomy for node-positive breast cancer. J Clin Oncol. 1990; 8 (8): 1327-34.

Chlebowski RT, Aiello E, McTiernan A. Weight loss in breast cancer patient management. J Clin Oncol. 2002; 20: 1128-43.

Chlebowski RT, Blackburn GL, Thomson CA, Nixon DW, Shapiro A, Hoy MK, et al. Dietary fat reduction and breast cancer outcome: interim efficacy results from women's intervention nutrition study. J Natl Cancer Inst. 2006; 98: 1767-76. 
Clarke CA, Purdie DM, Glaser SL. Population attributable risk of breast cancer in white women associated with immediately modifiable risk factors. BMC Cancer. 2006; 6: 170-80.

Costa LJM, Varella PCS, Del Giglio A. Weight changes during chemotherapy for breast cancer. São Paulo Med J. 2002; 120 (4): 113-7.

Del Giglio A e Samano EST. Principais substâncias terapêuticas contra o câncer. In: Waitzberg DL. Dieta, nutrição e câncer. São Paulo: Atheneu; 2004. p. 123-38.

Del Rio G, Zironi S, Valeriani L, Menozzi R, Bondi M, Bertolini M, et al. Weight gain in women with breast cancer treated with adjuvant cyclophosphomide, methotrexate and 5-fluorouracil. Analysis of resting energy expenditure and body composition. Breast Cancer Res Treat. 2002; 73 (3): 267-73.

Demark-Wahnefried W, Kenyon AJ, Eberle P, Skye A, Kraus WE. Preventing sarcopenic obesity among breast cancer patients who receive adjuvant chemotherapy: results of feasibility study. Clin Exerc Physiol. 2002; 4 (1): 44-9.

Demark-Wahnefried W, Peterson B, McBride C, Lipkus I, Clipp E. Current health behaviors and readiness to pursue life-style changes among men and women diagnosed with early stage prostate and breast carcinomas. Cancer. 2000; 88: 674-84.

Demark-Wahnefried W, Peterson BL, Winer EP, Marks L, Aziz N, Marcom PK, et al. Changes in weight, body composition, and factors influencing energy balance among premenopausal breast cancer patients receiving adjuvant chemotherapy. J Clin Oncol. 2001; 19 (9): 2381-89.

Demark-Wahnefried W, Rimer BK, Winer EP. Weight gain in women diagnosed with breast cancer. J Am Diet Assoc. 1997; 97 (5): 519-26.

Demark-Wahnefried W, Winer EP e Rimer BK. Why women gain weight with adjuvant chemotherapy for breast cancer. J Clin Oncol. 1993; 11: 1418-29.

Doyle C, Kushi LH, Byers T, Courneya KS, Demark-Wahnefried W, Grant B, et al. Nutrition and physical activity during and after cancer treatment: an American Cancer Society guide for informed choices. CA Cancer J Clin. 2006; 56: 323-53.

Freedman RJ, Aziz N, Albanes D, Hartman T, Danforth D, Hill S, et al. Weight and body composition changes during and after adjuvant chemotherapy in women with breast cancer. J Clin Endocrinol Metab. 2004; 89: 2248-53. 
Gimenes DL. Quimioterapia. In: Ikemori EH, Oliveira T, Serralheiro IFD, Shibuya E, Cotrim TH, Trintin LA, et al. Nutrição em oncologia. São Paulo: Lemar Livraria; Editora Marina e Tecmedd Editora; 2003. p. 179-88.

Goodwin PJ, Ennis M, Pritchard KI, Koo J, Trudeau ME, Hood N. Diet and breast cancer: evidence that extremes in diet are associated with poor survival. J Clin Oncol. 2003; 21: 2500-07.

Goodwin PJ, Ennis M, Pritchard KI, McCready D, Koo J, Sidiofsky S, et al. Adjuvant treatment and onset of menopause predict weight gain after breast cancer diagnosis. J Clin Oncol. 1999; 17 (1): 120-29

Harvie MN, Campbell IT, Baildam A, Howell A. Energy balance in early breast cancer patients receiving adjuvant chemotherapy. Breast Cancer Res Treat. 2004; 83 (3): 201-10.

Holick CN, Newcomb AP, Trentham-Dietz A, Titus-Ernstoff L, Bersch AJ, Stampfer MJ, et al. Physical activity and survival after diagnosis of invasive breast cancer. Cancer Epidemiol Biomarkers Prev. 2008; 17 (2): 379-86.

Holmes MD, Chen WY, Feskanich D, Kroenke CH, Colditz GA. Physical activity and survival after breast cancer diagnosis. JAMA. 2005; 293: 2479-86.

Holmes MD, Stampfer MJ, Colditz GA, Rosner B, Hunter DJ, Willet WC. Dietary factors and survival of women with breast carcinoma. Cancer. 1999; 86: 826-35.

Howard BV, Manson JE, Stefanick ML, Beresford SA, Frank G, Jones B, et al. Low-fat dietary pattern and weight change over 7 years. JAMA. 2006; 295: 3949.

INCA - Instituto Nacional de Câncer. A situação do câncer no Brasil. Rio de Janeiro, 2006.

INCA - Instituto Nacional de Câncer. Estimativa 2008: Incidência de câncer no Brasil. Rio de Janeiro, 2007.

INCA - Instituto Nacional de Câncer. Coordenação de Prevenção e Vigilância (CONPREV). Controle do câncer de mama. Documento de consenso. Rio de Janeiro, 2004a.

INCA - Instituto Nacional de Câncer. TNM: classificação de tumores malignos / traduzido por Ana Lúcia Amaral Eisenberg. 6. ed. Rio de Janeiro, 2004b. 
Ingram C, Brown JK. Patterns of weight and body composition change in premenopausal women with early stage breast cancer. Has weight gain been overestimated? Cancer Nursing ${ }^{\mathrm{TM}}$. 2004; 27 (6): 483-90.

Irwin ML. Physical activity interventions for cancer survivors. Br J Sports Med. 2009; 43: 32-8.

Irwin ML, Crumley D, McTiernan A, Bernstein L, Baumgartner R, Gilliland FD, et al. Physical activity levels before and after a diagnosis of breast carcinoma. Cancer. 2003; 97: 1746-57.

Irwin ML, McTiernan A, Baumgartner RN, Baumgartner KB, Bernstein L, Gilliland FD, et al. Changes in body fat and weight after a breast cancer diagnosis: influence of demographic, prognostic, and lifestyle factors. J Clin Oncol. 2005; 23 (4): 774-82.

Irwin ML, McTiernan A, Bernstein L, Gilliland FD, Baumgartner R, Baumgartner $\mathrm{K}$, et al. Physical activity levels among breast cancer survivors. Med Sci Sports Exerc. 2004; 36: 1484-91.

Kelsey JL e Berkowitz, GS. Breast Cancer Epidemiology. Cancer Research. 1988; 48 (15): 5615-23.

Kwan ML, Weltzien E, Kushi LH, Castillo A, Slattery ML, Caan BJ. Dietary patterns and breast cancer recurrence and survival among women with earlystage breast cancer. J Clin Oncol. 2008; 27: 919-26.

Kirjner A e Pinheiro RL. Interferência da obesidade no tratamento quimioterápico em mulheres com câncer de mama. Revista Brasileira de Cancerologia. 2007; 53 (3): 345-54.

Kroenke $\mathrm{CH}$, Fung TT, Hu FB, Holmes MD. Dietary patterns and survival after breast cancer diagnosis. J Clin Oncol. 2005; 23: 9295-303.

Kumar N, Allen KA, Riccardi D, Bercu BB, Cantor A, Minton S, et al. Fatigue, weight gain, lethargy and amenorrhea in breast cancer patients on chemotherapy: is subclinical hypothyroidism the culprit? Breast Cancer Res Treat. 2004; 83 (2): 149-59.

Kushi LH, Kwan ML, Lee MM, Ambrosone CB. Lifestyle factors and survival in women with breast cancer. J. Nutr. 2007; 137: 236S-42S. 
Kutynec CL, McCargar L, Barr SI, Hislop G. Energy balance in women with breast cancer during adjuvant treatment. J Am Diet Assoc. 1999; 99 (10): 122227.

Lankester KJ, Phillips JE, Lawton PA. Weight gain during adjuvant and neoadjuvant chemotherapy for breast cancer: an audit of 100 women receiving FEC or CMF chemotherapy. Clinical Oncology. 2002; 14: 64-7.

Lima-Junior JAT, Pinto-Neto AM, Costa-Paiva LHS, Pedro AO. Variação no índice de massa corporal em usuárias de terapia de reposição hormonal. RBGO. 2000; 22 (4): 227-31.

Loi S, Milne RL, Friedlander ML, McCredie MRE, Giles GG, Hopper JL. Obesity and outcomes in premenopausal and postmenopausal breast cancer. Cancer Epidemiol Biomarkers Prev. 2005; 14 (7): 1686-91.

Loprinzi CL, Athmann LM, Kardinal CG, O'Fallon JR, See JA, Bruce BK, et al. Randomized trial of dietician counseling to try to prevent weight gain associated with breast cancer adjuvant chemotherapy. Oncology. 1996; 53: 228-32.

Malzyner A, Caponero R. Consequências nutricionais do tratamento quimioterápico. In: Waitzberg DL. Dieta, nutrição e câncer. São Paulo: Atheneu; 2004. p. 399-406.

Maraki-Judson G, Judson CH, Mertens WC. Longitudinal patterns of weight gain after breast cancer diagnosis: observations beyond the first year. Breast Journal. 2007; 13 (3): 258-65.

Maunsell E, Drolet M, Brisson J, Robert J, Deschênes L. Dietary change after breast cancer: extent, predictors, and relation with psychological distress. J Clin Oncol. 2002; 20: 1017-25.

Mc Eligot AJ, Largent J, Ziogas A, Peel D, Anton-Culver H. Dietary fat, fiber, vegetable, and micronutrients are associated with overall survival in postmenopausal women diagnosed with breast cancer. Nutrition and Cancer. 2006; 55 (2): 132-40.

McTiernan A, Kumar BR, Tworoger SS, Irwin M, Bernstein L, Baumgartner R, et al. Adiposity and sex hormones in postmenopausal breast cancer survivors. J Clin Oncol. 2003; 21: 1961-66.

OMS - Organização Mundial da Saúde. Physical Status: the use and interpretation of anthropometry. Technical Report Series, 854. Genebra; 1995. 
OPAS - Organização Pan-Americana de Saúde. XXXVI Reunión Del Comitê Asesor de Investigaciones em Salud - Encuesta Multicêntrica - Salud, Bienestar y Envejecimiento (SABE) en América Latina e el Caribe - Informe preliminar. 2001.

Paulinelle RR, Freitas Jr R, Curado, MP, Souza AA. A situação do câncer de mama em Goiás, no Brasil e no mundo: tendências atuais para a incidência e a mortalidade. Rev Bras Saúde Mater Infant. 2003; 3 (1): 17-24.

Pierce JP, Natarajan L, Caan BJ, Parker BA, Greenberg ER, Flatt SW, et al. Influence of a diet very high in vegetables, fruit, and fiber and low in fat on prognosis following treatment for breast cancer: the women's healthy eating and living (WHEL) randomized trial. JAMA. 2007; 298 (3): 289-98.

Pinder MC, Duan Z, Goodwin JS, Hortobagyi GN, Giordano SH. Congestive heart failure in older women treated with adjuvant anthracycline chemotherapy for breast cancer. J Clin Oncol 2007; 25: 3808-15.

Pinto BM e Trunzo JJ. Body esteem and mood among sedentary and active breast cancer survivors. Mayo Clin Proc. 2004; 79: 181-86.

Poehlman ET e Tchernof A. Transversing the menopause: changes in energy expenditure and body composition. Coron Artery Dis. 1998; 9(12):799-803.

Rock CL. Diet and breast cancer: can dietary factors influence survival? Journal of Mammary Gland Biology and Neoplasia. 2003; 8 (1): 119-32.

Rock CL e Demark-Wahnefried W. Nutrition and survival after the diagnosis of breast cancer: a review of evidence. J Clin Oncol. 2002; 20 (15): 3302-16.

Rock CL, Flatt SW, Newman V, Caan BJ, Haan MN, Stefanick ML, et al. Factors associated with weight gain in women after diagnosis of breast cancer. J Am Diet Assoc. 1999; 99 (10): 1212-18.

Rock CL, Flatt SW, Thomson CA, Stefanick ML, Newman VA, Jones LA, et al. Effects of a high-fiber, low-fat diet intervention on serum concentrations of reproductive steroid hormones in women with a history of breast cancer. J Clin Oncol. 2004; 22 (12): 2379-87.

Rock CL, Thomson C, Caan BJ, Flatt SW, Newman V, Ritenbaugh C, et al. Reduction in fat intake is not associated with weight loss in most women after breast cancer diagnosis. Evidence from a randomized controlled trial. Cancer. 2001; 91: 25-34. 
Rooney $\mathrm{M}$ e Wald $\mathrm{A}$. Interventions for the management of weight and body composition changes in women with breast cancer. Clin J Oncol Nurs. 2007; 11 (1): 41-52.

Schmitt F e Gobbi H. Mama. In: Brasileiro Filho G. Bogliolo Patologia. $7^{\text {a }}$ Ed. Rio de Janeiro: Guanabara Koogan; 2006. p. 613-43.

Schwartz AL. Exercise and weight gai in breast cancer patients receiving chemotherapy. Cancer Practice. 2000; 8 (5): 231-7.

Tao M-H, Shu X-O, Ruan ZX, Gao Y-T, Zheng W. Association of overweight with breast cancer survival. Am J Epidemiol. 2006; 163: 101-7.

Thomson CA, Rock CL, Giuliano AR, Newton TR, Cui H, Reid PM. Longitudinal changes in body weight and body composition among women previously treated for breast cancer consuming a high-vegetable, fruit and fiber, low-fat diet. Eur J Nutr. 2005; 44: 18-25.

Thuler LC. Considerações sobre a prevenção do câncer de mama feminino. Revista Brasileira de Cancerologia. 2003; 49 (4): 227-38.

Wu AH, Pike MC, Stram DO. Meta-analysis: dietary fat intake, serum estrogen levels, and the risk of breast cancer. J Natl Cancer Inst. 1999; 91: 529-34. 


\section{ANEXO 1 - TERMO DE CONSENTIMENTO LIVRE E ESCLARECIDO}

Resolução nº 196, de 10 de Outubro de 1996, segundo o Conselho Nacional de Saúde

\section{Dados de Identificação \\ Nome da Paciente:}

\section{I - DADOS DE IDENTIFICAÇÃO DO SUJEITO DA PESQUISA}

RG n:

Data de Nascimento: I .$/$ Telefone:

Endereço:

Cidade: No:

CEP:

Apto:

Bairro: Sexo: ( ) M ( ) F
Telefone: ( )

2. Responsável

Legal:

Natureza (grau de parentesco, tutor, curador, etc.)

RG $n^{\circ}$ :

Data de Nasc

Bairro:

Cidade:

$\mathrm{N}^{\mathrm{O}}$ :

Apto:

CEP :

\section{II- DADOS SOBRE A PESQUISA}

1. Título do Protocolo de Pesquisa: Aumento de peso em mulheres com câncer de mama e associação com quimioterapia e recidiva da doença.

Pesquisador: Maria de Fátima Nunes Marucci
RG n : 4.791.628-X
Sexo: ( ) M
(x) $\mathrm{F}$

Cargo / Função: Professora Doutora

Departamento de Nutrição da Faculdade de Saúde Pública

\section{Avaliação de Risco da Pesquisa}
(x) Sem Risco
( ) Risco Mínimo
( ) Risco Médio
( ) Risco Baixo
Risco Maior

()

3. Duração da Coleta de dados: 3 a 4 meses

\section{III - REGISTRO DAS EXPLICAÇÕES DO PESQUISADOR AO SUJEITO DA PESQUISA OU SEU REPRESENTANTE LEGAL SOBRE A PESQUISA, CONSIGNANDO}

2. Justificativa e Objetivos: Este projeto pretende verificar o aumento de peso corporal e os efeitos da quimioterapia e a recidiva da doença, em mulheres com câncer de mama. Por isso, estou convidando-a para participar desta pesquisa e, preciso conhecer a evolução da doença. Para tanto, necessito consultar o seu prontuário, a fim de coletar dados específicos como: idade, na ocasião do diagnóstico, valores de peso, no início e final do tratamento, situação em relação aos ciclos menstruais, fase da doença, tratamento e número de ciclos da quimioterapia, fatores hormonais e envolvimento de linfonodos. Além disso, gostaria de saber se houve alteração do consumo de alimentos e na prática de atividade física, para mais ou para menos, durante o tratamento.

3. Procedimentos que serão utilizados e propósitos: Coleta de dados em prontuário

4. Desconfortos e riscos esperados: Não se aplica.

5. Benefícios que poderão ser obtidos: Melhor orientação das mulheres com câncer de mama, quanto aos cuidados necessários em relação ao aumento de peso e à recidiva da doença e melhor controle do quadro clínico. 


\section{IV - ESCLARECIMENTOS DADOS PELO PESQUISADOR SOBRE GARANTIAS DA PARTICIPANTE DA PESQUISA}

1. A senhora terá acesso, em qualquer momento, às informações sobre procedimentos, riscos e benefícios relacionados à pesquisa, inclusive para esclarecer qualquer dúvida;

2. A senhora poderá retirar seu consentimento a qualquer momento e deixar de participar do estudo, sem que isto traga prejuízo à continuidade da assistência médica;

3. A senhora terá a garantia da confidencialidade, sigilo e privacidade, uma vez que seu nome não será divulgado na pesquisa ou em qualquer outro local. Entretanto, os resultados da pesquisa poderão ser apresentados em congressos, reuniões científicas e publicados em revistas científicas.

\section{IV - INFORMAÇÕES DE NOMES, ENDEREÇOS E TELEFONES DOS RESPONSÁVEIS PELO ACOMPANHAMENTO DA PESQUISA PARA CONTATO EM CASO DE INTERCORRÊNCIAS CLÍNICAS E REAÇÕES ADVERSAS}

1. PESQUISADORA EXECUTANTE: Eliana da Silva Rezende Mendes

Rua: Dankmar Adler, 100, ap. 92, Morumbi, São Paulo/SP - Telefones: 3772-7180 ou 9606-4541 e-mail: elianamendes@usp.br

2. PESQUISADORA RESPONSÁVEL: Profa. Dra. Maria de Fátima Nunes Marucci

Av. Dr. Arnaldo, 715, Cerqueira César, São Paulo/SP

3. CLIOH: Av. Rebouças, 3387, Pinheiros, São Paulo/SP - Telefones: 3819-5007 ou 3819-2015 e-mail: clioh@clioh.com.br

Declaro que, após ter sido suficientemente esclarecida pela pesquisadora e ter entendido o que me foi explicado, concordo em participar da presente pesquisa.

São Paulo, de de

\footnotetext{
Assinatura do sujeito de pesquisa
} ou responsável legal

Assinatura do pesquisador (carimbo ou nome legível) 


\section{ANEXO 2 - FICHA PARA COLETA DOS DADOS}

\section{I - DADOS DEMOGRÁFICOS}

Nome:

Idade no momento do diagnóstico:

Data de Nascimento:

\section{II - DADOS CLÍNICOS}

Estadiamento tumoral:

"Status" dos receptores hormonais: ( ) ER+ / PR+ ( ) ER-/PR+ ( ) ER+/PR- ( ) ER-/PR-

Número de linfonodos comprometidos:

Fase do climatério: ( ) pré-menopausa ( ) pós-menopausa

Protocolo quimioterápico:
( ) com antraciclina: ( ) AC
( ) FAC
( ) FEC

( ) sem antraciclina (CMF)

Número de ciclos: ( ) 4 ciclos ( ) 6 ciclos

Peso inicial: $\mathrm{kg}$

Peso final: $\mathrm{kg}$

Ocorrência de recidiva da doença: ( ) sim Em quanto tempo?

( ) não

Durante a quimioterapia, a Sra. teve alteração de consumo de alimentos?

( ) Sim ( ) Não

Em caso afirmativo: ( ) Aumentou ( ) Diminuiu

Durante a quimioterapia, a Sra. teve alteração na prática de atividade física?

( ) Sim ( ) Não

Em caso afirmativo: ( ) Aumentou ( ) Diminuiu 


\title{
ANEXO 3 - PARECER DO COMITÊ DE ÉTICA
}

\author{
Universidade de São Paulo
COMITE DE ÉTICA EM PESQUISA - COEP/FSP \\ Faculdade de Saúde Pública \\ Of.COEP/ $50 / 08$
}

$\begin{array}{ll}\text { Protocolo } & 1751 \\ & \\ \text { Projeto de Pesquisa } & \text { AUMENTO DE PESO EM MULHERES COM CÂNCER DE MAMA E } \\ \text { Pesquisador(a) } & \text { Eliana da Silva Rezende Mendes }\end{array}$

26 de MARÇO de 2008

Prezado(a) Orientador(a),

O Comitê de Ética em Pesquisa da Faculdade de Saúde Pública da Universidade de São Paulo - COEP analisou, em sua 2. \%/08 Sessão ORDINÁRIA, realizada em 20/03/2008, de acordo com os requisitos da Resolução CNS/196/96 e suas complementares, o protocolo de pesquisa acima intitulado e o considerou APROVADO.

Cabe lembrar que conforme Resolução CNS/196/96 são deveres do (a) pesquisador (a): 1. Comunicar, de imediato, qualquer alteração no projeto e aguardar manifestação deste CEP (Comitê de Ética em Pesquisa), para dar continuidade à pesquisa;

2. Manter sob sua guarda e em local seguro, pelo prazo de 5 (cinco) anos, os dados da pesquisa, contendo fichas individuais e todos os demais documentos recomendados pelo CEP, no caso eventual auditoria;

3. Comunicar, formalmente a este Comitê, quando do encerramento deste projeto;

4. Elaborar e apresentar relatórios parciais e finais;

5. Justificar, perante o CEP, interrupção do projeto ou a não publicação dos resultados.

Atenciosamente,

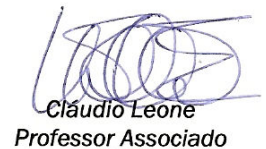

Professor Associado

Coordenador do Comitê de Ética em Pesquisa - FSP/COEP

IIm. ${ }^{a} \mathrm{Sr}^{\mathrm{a}}$

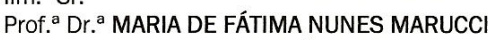

DEPARTAMENTO DE NUTRIÇÃO

Av. Dr. Arnaldo, 715 - Assessoria Acadêmica - CEP: 01246-904 - São Paulo - SP Telefones: (55-11) 3061-7779/7742 e-mail: coep@fsp.usp.br site www.fsp.usp.br 